\title{
Les isotopes du milieu dans les eaux naturelles
}

\author{
PAR
}

\author{
J.-C. Fontes \\ Laboratoire de géologie dynamique. \\ Université Pierre et Marie Curie, Paris
}

\section{Deux voies de traçage}

Les fondements de la technique du traçage en Hydrologie sont simples: repérage, et le plus souvent mesure des teneurs en composés chimiques, en substances colorées, en molécules isotopiques stables ou radioactives, ou même en produits solides figurés, présents en un point du cycle hydrologique placé sous étude.

Le traçage artificiel consiste à introduire des substances déterminées dans le milieu où elles étaient absentes ou très faiblement représentées. Le traçage naturel se limite à l'examen des teneurs présentes dans le milieu sans opération d'ensemencement.

Le traçage artificiel n'est point commenté ici. Soulignons simplement que des groupes spécialisés, comme celui du Centre d'Etudes Nucléaires de Grenoble, l'équipe de l'Institut des Mines de Cracovie, ont largement contribué à définir les domaines d'emploi et les lois d'exploitation des informations livrées par les traceurs artificiels en Hydrologie.

On retiendra simplement que certains facteurs intrinsèques (coût, niveau de détection, maitrise simultanée de l'injection et du rejet) rendent l'usage des traceurs artificiels particulièrement adapté aux phénomènes hydrologiques à pas de temps brefs ou à mailles spatiales courtes. Bien souvent, l'efficacité du traceur artificiel s'impose élégamment dans les études d'eaux de surface, et de circulations souterraines rapides (karsts ou roches largement fissurées, nappes libres parfois).

Le traçage naturel, ou mieux traçage du milieu si l'on suit les recommandations de la Décennie hydrologique de l'UNES$\mathrm{CO}$, recouvre théoriquement toutes les approches de type hydrochimique. Les avantages, qu'indiquent la méthodologie, résident essentiellement dans la possibilité de s'adresser à un milieu quelconque, sans pratiquement encourir aucune limitation imputable aux dimensions ou aux durées des objets d'étude. Autre particularité importante et d'intérêt : les traceurs du milieu sont généralement en régime permanent en chaque point de leurs cycles, y compris dans leurs fluctuations de teneur. Parmi ces marqueurs du milieu une place spéciale est accordée aux traceurs isotopiques du milieu (Environmental isotopes de la littérature anglo-saxonne).

\section{Isotopes du milieu : rappels de quelques principes.}

Les isotopes du milieu mettent en oeuvre des lois de répartition identiques à celles qui régissent les réactions chimiques : lois d'action de masse appliquées aux activités ou aux fugacités des molécules isotopiques. On ajoutera cependant une spécifité thermodynamique des isotopes. Ils peuvent s'échanger entre deux composés ou deux phases comportant le même élément sans qu'aucune réaction, autre qu'isotopique, ne s'opère d'une phase à l'autre, d'un composé à l'autre.

Par exemple, les réactions isotopiques suivantes sont, globalement, la règle sans qu'intervienne d'autre transfert de masse qu'un échange, très limité au demeurant $\left({ }^{1}\right)$, des différentes espèces isotopiques :

$$
\begin{gathered}
\mathrm{H}_{2}{ }^{18} \mathrm{O}+\frac{1}{2} \mathrm{C}^{16} \mathrm{O}_{2} \rightleftarrows \mathrm{H}_{2}{ }^{16} \mathrm{O}+\frac{1}{2} \mathrm{C}^{18} \mathrm{O}_{2} \\
\mathrm{H}_{2}{ }^{16} \mathrm{O} \text { liquide }+\mathrm{H}_{2}{ }^{18} \mathrm{O} \text { vapeur }
\end{gathered}
$$

(1) Dans la gamme des températures biologiques, les échanges isotopiques sont, sauf exception pour les composés hydrogénés, de quelques parts pour cent (parts de différences relatives du rapport isotopique). 
Un autre caractère distinctif des isotopes est, celui là, évident : les dispositifs de mesures ou de détection sont nécessairement différents des techniques chimiques ordinaires puisque, par définition, les isotopes ne répondent pas aux critères de distinction chimiques. On mesurera les très légères différences de masses dans le cas des isotopes stables (spectrométrie de masse), on enregistrera l'émission radioactive caractéristique (spectrométrie alpha ou gamma) ou la radioactivité totale (comptage béta) dans le cas des noyaux radioactifs.

Les isotopes stables font le plus souvent l'objet de mesures différentielles car leurs variations relatives sont suffisamment indicatives. On écrit alors, à l'aide de la notation très générale delta $(\delta)$ :

$$
\delta=\frac{R \text { échantillon }}{R \text { étalon }}-1
$$

avec $R=$ rapport isotopique $\approx$ concentration de l'espèce isotopique rare. Soit, par exemple :

$$
R=\frac{{ }^{18} \mathrm{O}}{{ }^{16} \mathrm{O}}
$$

Dans le cas des eaux, l'étalon est réputé représenter la composition isotopique moyenne des eaux océaniques: "Standard Mean Ocean Water": SM.O.W.(Craig, 1961 a). C"est un étalon logique et naturel puisque tous les cycles hydrologiques prennent leur source et font finalement retour à l'océan qui représente 97 à $98 \%$ de l'eau de l'hydrosphère.

Dans la pratique il est commode de traiter des chiffres simples et $\delta$ s'exprime en parts pour mille : par exemple :

$$
\delta=\left[\frac{{ }^{18} \mathrm{O} /{ }^{16} \mathrm{O} \text { éch } .}{{ }^{18} \mathrm{O} /{ }^{16} \mathrm{O} \text { étalon }}-1\right] 1000
$$

Selon les rapports isotopiques envisagés. l'incertitude sur $\delta$ s'inscrit généralement entre $\pm 0,05 \%$ et $\pm 0,3 \%$ lorsqu'elle est définie comme l'écart quadratique moyen sur une population suffisante de mesures.

Si l'on veut bien considérer, par exemple, que l'oxygène 18 qui dans les eaux est représenté en moyenne à raison de 2 atomes pour 1000 atomes d'oxygène 16 , se mesure relativement à environ $0.1 \%$, cela signifie que le spectromètre de masse autorise une détection meilleure que $10^{-6}$. Rap- portée à la plage des variations naturelles des teneurs en oxygène 18 (environ $100 \%$ ) c'est en définitive un traceur dont la sensibilité relative est proche de $10^{-9}$ soit encore 1000 à 10000 fois moins que les meilleurs traceurs artificiels mais plusieurs ordres de grandeur au-dessus de la sensibilite des analyses chimiques d'éléments majeurs et d'oligoéléments. Il en va sensiblement de mème pour les autres isotopes stables utilisés en hydrologie (tableau I)

Dans le cas des noyaux radioactifs, les sensibilités sont multipliées par plusieurs ordres de grandeur. Il est possible que le tritium, dont la production naturelle équilibrée par la décroissance, conduit à une teneur de l'ordre de 1 atome de ${ }^{3} \mathrm{H}$ pour $10^{17}$ atomes de ${ }^{1} \mathrm{H}$, n'aurait pas été découvert s'il n'avait été émetteur béta.

Aussi bien le carbone 14 que le tritium ne doivent pas leur présence dans le milieu au seul impact de la composante neutronique du rayonnement cosmique sur les noyaux d'azote de l'air. Ils ont été également injectés en grande quantité dạn la stratosphère lors des essais thermonucléaires et nucléaires aériens. Leur concentration a donc varié largement au cours de ces vingt dernières années, avec un maximum lors des essais records des années 1963-1964 et une décroissance marquée depuis que la plupart des Nations ont renoncé aux essais aériens.

Les teneurs en tritium se repèrent en unités tritium (1 UT correspondant à 1 atome ${ }^{3} \mathrm{H}$ pour $10^{18}$ atomes ${ }^{1} \mathrm{H}$ ). Les activités en ${ }^{14} \mathrm{C}$ se définissent par rapport à la teneur dite "moderne" en pour cents. La teneur "moderne" ou "carbone moderne" est censée représenter l'activité du $\mathrm{CO}_{2}$ de l'atmosphère avant la contamination nucléaire d'une part et avant la pollution par les résidus de combustion de l'ère industrielle (effet Suess) d'autre part. Le premier type de pollution apporte un excès de carbone 14 , le second type, qui fait intervenir de très vieux carbones, conduit à une dilution $\mathrm{du}{ }^{14} \mathrm{C}$ de l'atmosphère. Par convention, on adopte l'année 1950 comme une date de référence où ces deux effets se sont équilibrés. L'activité en ${ }^{14} \mathrm{C}$ sera repérée en pour cent de carbone moderne, l'activité de $100 \%$ étant celle du $\mathrm{CO}_{2}$ de l'atmosphère en 1950 (et avant le début de l'ère industrielle, soit la première moitiè $\mathrm{du} \mathrm{XIX}^{\mathrm{e}}$ siècle).

Il faut souligner que les incertitudes sur les mesures de radioactivité sont fonction croissante de la racine carrée de l'activité enregistrée et que les incertitudes relatives diminuent donc lorsque l'activité augmente.

Tableau 1

\begin{tabular}{|c|c|c|c|c|c|}
\hline \multicolumn{2}{|c|}{$\begin{array}{c}\text { Elément et isotope } \\
\text { banal }\end{array}$} & Isotopes rares & $\begin{array}{c}\text { Fréquence moyenne } \\
\text { isotopes rares ppm }\end{array}$ & Formes étudiées & $\begin{array}{l}\text { Domaine de } \\
\text { variation } \%\end{array}$ \\
\hline \multirow{2}{*}{\multicolumn{2}{|c|}{ Hydrogène ${ }^{1} \mathrm{H}$}} & Deuterium : ${ }^{2} \mathrm{H}$ & 156 & eau, hydrogène, & 500 \\
\hline & & Tritum $\quad:{ }^{3} \mathrm{H}^{*}$ & $10^{-10}$ & hydrocarbures & $10^{-15} \quad 10^{-11}$ \\
\hline Oxygène & ${ }^{16} \mathrm{O}$ & Oxygène $18:{ }^{18} \mathrm{O}$ & 2000 & $\begin{array}{l}\text { eau, anions oxygénés, } \\
\mathrm{CO}_{2}\end{array}$ & 100 \\
\hline \multirow[t]{2}{*}{ Carbone } & ${ }^{12} \mathrm{C}$ & Carbone $13:{ }^{13} \mathrm{C}$ & $1,11 \cdot 10^{2}$ & $\begin{array}{l}\text { carbonates, } \mathrm{CO}_{2} \\
\text { hydrocarbures }\end{array}$ & 80 \\
\hline & & Carbone $14:{ }^{14} \mathrm{C}^{*}$ & $10^{-6}$ & $-d^{\circ}-$ & $10^{-7}$ à $10^{-9}$ \\
\hline Azote & ${ }^{14} \mathrm{~N}$ & Azote $15:{ }^{15} \mathrm{~N}$ & 3660 & $\begin{array}{l}\text { azote, nitrates, sels } \\
\text { ammoniacaux }\end{array}$ & $80(?)$ \\
\hline Soufre & ${ }^{32} \mathrm{~S}$ & Soufre $34:{ }^{34} \mathrm{~S}$ & $4,22 \cdot 10^{4}$ & $\begin{array}{l}\text { soufre, sulfates, } \\
\text { soufre réduit }\end{array}$ & 100 \\
\hline
\end{tabular}

Isotopes du milieu adaptés aux études hydrologiques

${ }^{3} \mathrm{H}^{*},{ }^{14} \mathrm{C}^{*}$ noyaux radioactifs 
Il est commode de caractériser les éléments radioactifs par leur constante de désintégration radioactive, qui suffit à définir la décroissance, en désignant la proportion de noyaux actifs en désintégration à chaque instant au sein d'une masse active considérée comme un système clos (réservoir).

Dans la pratique des études, on a recours le plus souvent à la notion de période $\tau$ qui contient exactement la même information avec :

$$
\tau=\frac{\ln 2}{\gamma}
$$

et qui repère le temps au bout duquel la radioactivité a épuisé la moitié du réservoir en noyaux actifs initiaux au cours du phénomène únivoque et exponentiel avec le temps de décroissance radioactive.

Il est généralement vain de chercher à mesurer les activités après 8 à 10 périodes de décroissance. Les périodes respectives conditionneront l'usage des traceurs du milieu radioactif, de la même façon qu'elles limitent l'emploi des traceurs radioactifs artificiels; le tritium ( $\tau \simeq 12,3$ ans) verra son emploi limité aux cycles hydrologiques relativement courts. Le carbone 14 ( $\tau \simeq 5730$ ans) est intéressant pour l'examen des cycles longs, par exemple pour les nappes captives.

Dans son ensemble, la méthode du traçage implique une comparaison entre les entrées et les sorties au sein d'un système à étudier. La proposition est assez simple dans le cas du traçage artificiel. Pour ce qui regarde les isotopes du milieu. il est nécessaire de connaitre ou d'établir les fonctions entrćes. c'est-à-dire des lois ou les paramètres de variations de teneurs en isotopes du milieu dans les précipitations et les eaux de surface et de sub-surface.

\section{Les fonctions entrées des isotopes du milieu et leurs variations.}

\subsection{DEUX CATEGORIES FONDAMENTALES DE TRA- CEURS : TRACEURS INTERNES ET TRACEURS EXTERNES.}

Il est aisé d'admettre que le problème essentiel du traçage réside sinon dans l'identité du moins dans l'identification de comportement entre le traceur et son vecteur, l'eau. Les multiples réactions possibles, et bien souvent déjà recensées, au sein d'un milieu hétérogène, comme par exemple celui d'un aquifère (ad ou absorption, diffusion moléculaire et ionique, diffusion turbulente, réactions entre les sphères d'hydratation et le substrat, fractionnements ioniques au cours des effets d'ultrafiltration, échanges de base alcalins-alcalinoterreux, etc...) incitent à la prudence et souvent à la suspicion. Cependant on peut considérer de façon très schématique que les effets susceptibles d'introduire des variations de comportement entre l'eau et la substance traçante se résument à deux causes :

- différence de dimension :

- différences d'énergie potentielle disponibles aux interfaces eau-milieu, traceur-milieu, eau-traceur.

Certaines discussions récentes $\left({ }^{1}\right)$ nous fournissent l'opportunité de rappeler que les substances qui ont le plus de

(1) Selon D.R. Nielsen et G. Vachaud (Interventions orales au Colloque de Grenoble sur l'Ecoulement des fluides en milieux poreux C.N.R.S., Grenoble 1975) le tritium et l'oxygène 18 pourraient ne point être en toutes circonstances de "bons" traceurs de l'eau. Donnons volontiers acte à nos collègues du bien fondé de leur prudence. Si la zone non saturée se trouve par exemple portée a $400^{\circ} \mathrm{C}$ lors d'une éruption volcanique, les essais de traçage isotopique peuvent en effe se révéler peu significatifs (cf. 3.2.1.4). probabilité de se comporter de façon proche de celle de l'eau dans un milieu déterminé sont celles qui ont les dimensions et les densités d'électrons périphériques les plus voisines de celles de la molécule $\mathrm{H}_{2} \mathrm{O}$. Ceci nous impose d'introduire au sein de l'ensemble des traceurs une classe privilégiée : les espèces moléculaires de l'eau elle-même : soit en se bornant aux trois combinaisons qui présentent un intérêt pratique parmi toutes celles qu'offrent ${ }^{16} \mathrm{O},{ }^{17} \mathrm{O},{ }^{18} \mathrm{O},{ }^{1} \mathrm{H},{ }^{2} \mathrm{H},{ }^{3} \mathrm{H}$ :

$$
\left.\begin{array}{ll}
{ }^{1} \mathrm{H}_{2}{ }^{18} \mathrm{O} & : \text { Oxygène } 18 \\
{ }^{1} \mathrm{H}^{2} \mathrm{H}^{16} \mathrm{O} & : \text { Deuterium } \\
{ }^{1} \mathrm{H}^{3} \mathrm{H}^{16} \mathrm{O} & : \text { Tritium }
\end{array}\right\} \text { traceurs intimes }
$$

La molécule d'eau est tracée de l'intérieur, dans l'intimité de son noyau, c'est le traçage le plus fin et le plus conforme à l'objet tracé qui puisse à l'heure actuelle se concevoir.

Toutes les autres substances traçantes correspondent à un traçage externe aux édifices moléculaires ou ioniques et à leur cortège électronique.

Les causes de variations de teneur en traceurs intimes sont d'abord les lois générales de la physique de l'eau appliquée aux espèces isotopiques. Les traceurs externes verront leurs conditions d'entrée dans un système hydrologique conditionnées par les facteurs locaux.

\subsection{RAPPEL DES LOIS DE VARIATIONS DES CONCEN- TRATIONS EN ISOTOPES DE L'OXYGENE ET DE L'HYDROGENE, TRACEURS INTIMES DE L'EAU.}

\subsubsection{Isotopes stables : oxygène 18 et deuterium}

\subsubsection{Fractionnement isotopique lors des changements de phase.}

Les diverses molécules isotopiques ont des tensions de vapeur saturantes qui diffèrent très légèrement. Cela justifie l'apparition d'un fractionnement isotopique lors d'un changement partiel de phase par condensation ou vaporisation. De la même façon, les énergies libres de formation des molécules isotopiques solides et liquides étant différentes, il est normal d'attendre un autre fractionnement isotopique au passage partiel phase liquide-phase solide.

Ces fractionnements, prévus par le calcul théorique (Urey 1947, Bigeleisen, 1965), ont été expérimentalement reproduits (Bottinga et Craig, 1969, Merlivat et Nief, 1967, O'Neil, 1968) ainsi que leur dépendance vis-à-vis de la température.

D'une façon générale dans le domaine des températures des eaux de surface et de subsurface le fractionnement isotopique décroit lorsque la température augmente. Par exemple, pour l'oxygène 18 Bottinga et Craig (1969, op. cit.) proposent pour le passage liquide-vapeur l'équation empirique

$$
1000 \ln \alpha^{18} \mathrm{O}=2,644-3,206 \times 10^{3} / T+1,534 \times 10^{6} / T^{2}
$$

dans laquelle $T$ est la température en Kelvin et $\delta^{18} \mathrm{O}$ est le facteur de fractionnement :

$$
\alpha^{18} \mathrm{O}=\frac{\left({ }^{18} \mathrm{O} /{ }^{16} \mathrm{O}\right) \text { eau liquide }}{\left({ }^{18} \mathrm{O} /{ }^{16} \mathrm{O}\right) \text { eau vapeur }}
$$

Dans la pratique $\alpha$ est toujours peu différent de 1 et l'expression ln $\alpha$ est peu différente de $\alpha-1$. Elle traduit l'enrichissement (ou l'appauvrissement) isotopique de l'une des phases par rapport à l'autre. Mais l'important n'est point tellement que l'enrichissement isotopique qui sépare le liquide de la vapeur qui s'en échappe (à saturation) soit plus élevé à basse température. L'important est que, dans le do- 
maine des températures de l'hydrologie, cet enrichissement soit univoque. La vapeur est toujours appauvrie en isotopes lourds par rapport au liquide qui lui a donné naissance. A contrario le condensat est toujours enrichi en isotopes lourds au détriment de la phase vapeur (ou liquide) d'origine (fig. 1).

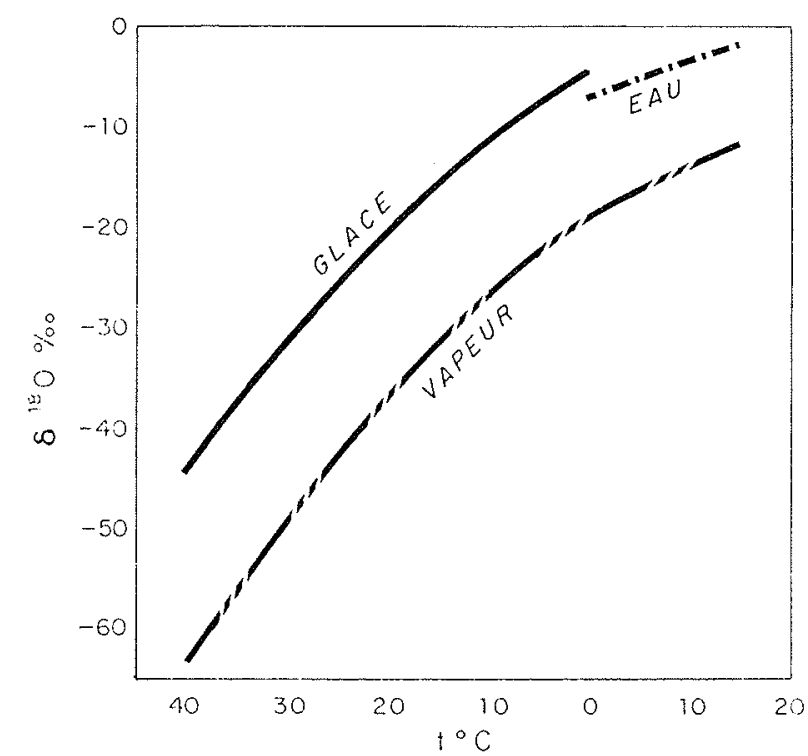

1/ Fractionnement isotopique

Fractionnement isotopique de l'oxygène en fonction de la température entre l'eau liquide et la vapeur au-dessus de zéro ${ }^{\circ} \mathrm{C}$ et entre l'eau solide et la vapeur au-dessous de zéro ${ }^{\circ} \mathrm{C}$. D'après R. Gonfiantini (1971).

\subsubsection{Traçage à la condensation.}

Tout épisode de précipitation appauvrit donc le réservoir de vapeur en isotopes lourds. Par la suite, cette masse de vapeur ne donnera lieu à de nouveaux stades de condensation que si la température s'abaisse. Le réservoir subit alors un appauvrissement supplémentaire tandis que la nouvelle phase condensée à partir d'une réserve d'isotopes lourds déjà amoindrie sera elle-même moins riche en ${ }^{18} \mathrm{O}$ et ${ }^{2} \mathrm{H}$ que lors de l'épisode précédent. Et ainsi de suite. On conçoit que la température de condensation et la composition isotopique des eaux météoriques soient liées. Là apparaît le principe du traçage naturel des précipitations. En effet, les profils thermiques de l'atmosphère sont généralement tels que l'on peut établir une relation de simple proportionnalité entre la température de condensation à la base du nuage et la température au sol. Bien entendu cela n'est vérifié de façon satisfaisante qu'à l'échelle de moyennes qui permettent d'émousser les effets des anomalies météorologiques qui accompagnent parfois les épisodes de précipitations (inversion de température, développement de cellules de circulations convectives, occlusion de front, etc. ..).

Dans la pratique cette propriété est appréciable car elle relie la composition isotopique des eaux météoriques à des paramètres géographiques ou paléogéographiques, à savoir :

- la latitude (fig. 2)

- l'altitude (fig. 3)

- la saison (fig. 4)

- la période climatique (dans le cas des eaux anciennes précipitées sous un climat qui n'a plus cours de nos jours, comme cela est par exemple le cas pour les eaux héritées des époques glaciaires).

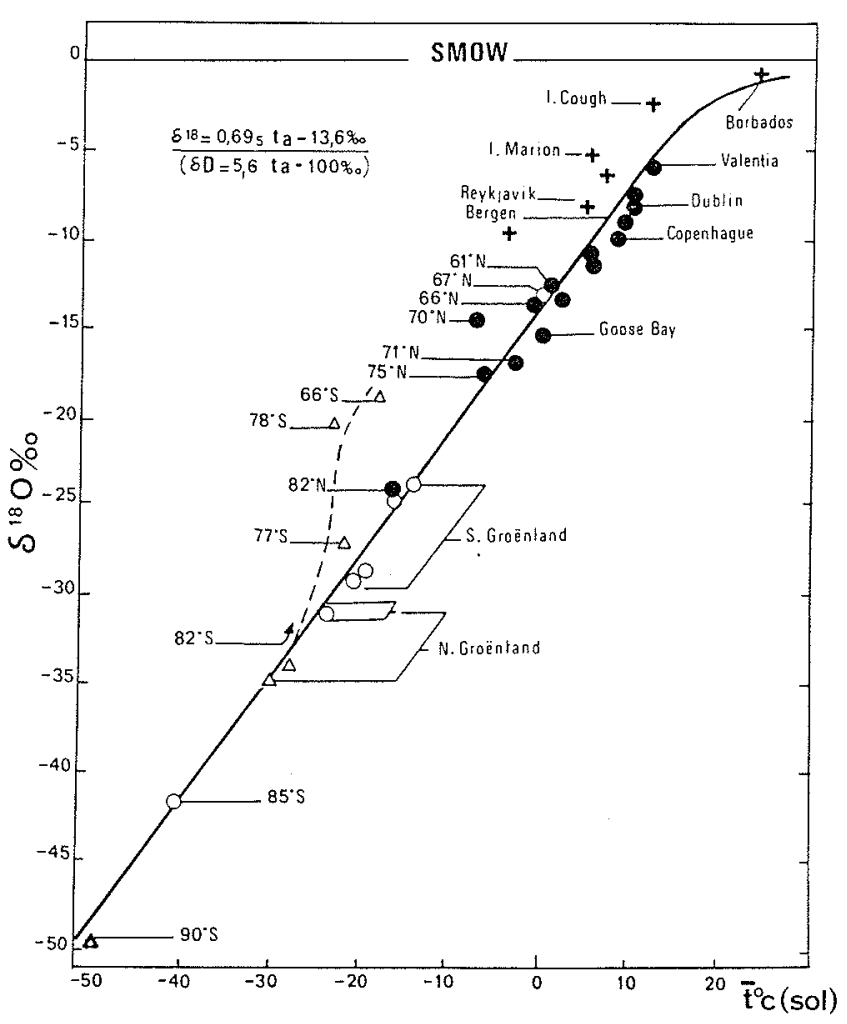

2/ Traçage des précipitations en isotopes stables.

Variation de la composition isotopique moyenne des précipitations $\left({ }^{18} \mathrm{O}\right)$ en fonction de la température annuelle moyenne au sol : traçage en latitude. Simplifié, d'après W. Dansgaard (1964).

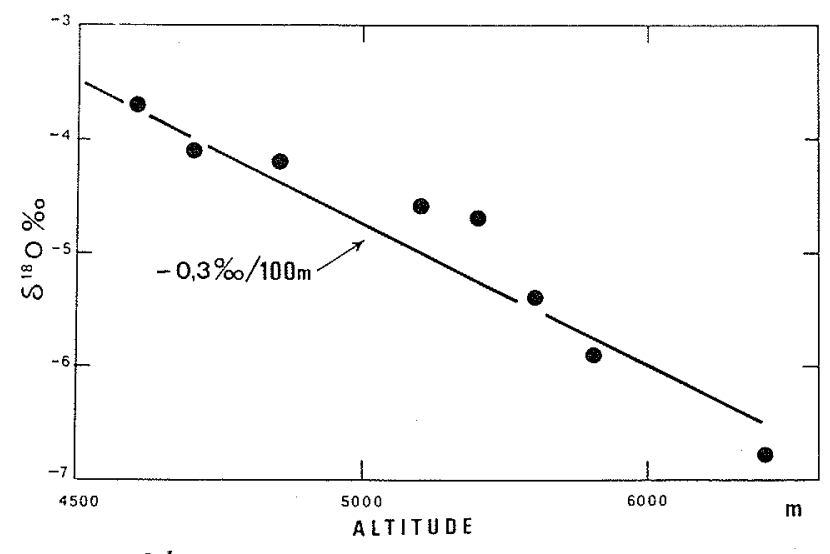

$3 /$ Traçage des précipitations en isotopes stables.

Variation de la teneur en ${ }^{18} \mathrm{O}$ d'un même épisode de précipitation en fonction de l'altitude. Il s'agit d'une collection de neige fraiche sur les pentes du Kilimandjaro. $D^{\prime}$ après E. Tongiorgi, inédit in R. Gonfiantini (1970).

Diverses tentatives ont été faites pour quantifier les relations de dépendance entre la température au sol et la teneur en isotopes lours des eaux précipitées.

A l'échelle mondiale, pour des stations océaniques généralement froides $(t$ moyen $<10 \mathrm{C}$ ) W. Dansgaard (1964) propose la relation :

$$
\delta^{18} 0=0,69 t_{\text {moyen annuel }}-13,6 \text { (fig. 2) }
$$

Cette relation ne s'applique plus dès que l'on considère une station n'obéissant plus à ce type relativement simple de condensation et en particulier les stations très continentales ou implantées dans des régions à forts reliefs. Il convient alors de rechercher les relations locales qui restent du même type. 


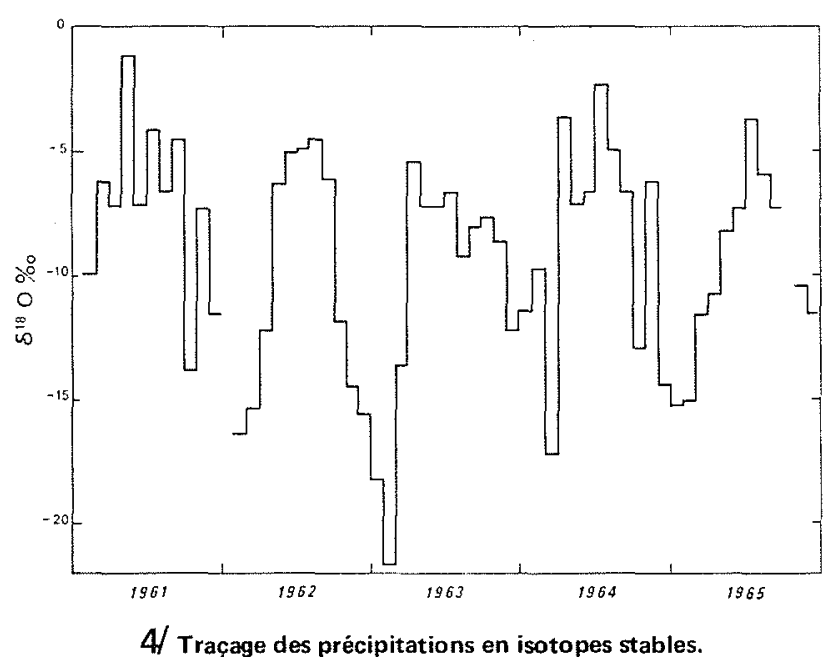

Variations saisonnières des teneurs en ${ }^{18} \mathrm{O}$ des précipitations à Vienne (Autriche). I.A.E.A. (1969-70-71-73).

Les variations de composition isotopique avec l'altitude sont évidemment différentes d'une localité à l'autre puisqu'elles ne font que traduire commodément les variations locales de température sur les versants. Les gradients cités dans la littérature varient de 0,16 à $0,70 \% / 100 \mathrm{~m}$. Les régions où la définition des profils est la plus détaillée indiquent $0,16 \% / 100 \mathrm{~m}$ au Mont Cameroun (Fontes et Olivry 1976), 0,26\% $/ 100 \mathrm{~m}$ sur la cote pacifique du Nicaragua (Payne et Yurtsever, 1974), à $0,35 \% / 100 \mathrm{~m}$ sur les versants de l'Appenin romain (Zuppi et al, 1974).

\subsubsection{Traçage à l'évaporation}

D'autre part, suite à la condensation, en cours de chute dans une zone désaturée de l'atmosphère, ou à la surface de sol avant l'infiltration et lors des épisodes de rétention, l'eau s'évapore. Cela se traduit par un enrichissement de la teneur en isotopes lourds de la fraction liquide restante.

Appauvrissement et enrichissement en isotopes lourds lors des phénomènes de condensation et d'évaporation n'atteignent point toutefois les valeurs que laissent prévoir l'application d'un simple modèle de distillation fractionnée (processus de Rayleigh) : la condensation parce que le nombre d'épisodes de distillation est forcement assez limité, l'évaporation parce qu'un phénomène complexe d'échange avec la vapeur atmosphérique ambiante vient limiter la variation de composition isotopique du liquide restant (Craig et al. 1963) : (fig. 5).

Les enrichissements en ${ }^{18} \mathrm{O}$ et en ${ }^{2} \mathrm{H}$ engendrés par l'évaporation ont un déterminisme complexe (Craig et Gordon, 1965 ; Gonfiantini, 1965 ; Fontes et Gonfiantini, 1967 ; Gat, 1970 ; Merlivat, 1970 ; Zimmerman et Ehhalt, 1970) qui fait intervenir la température, la teneur en eau de l'atmosphère, la vitesse d'évaporation ainsi que la diffusion différentielle des molécules isotopiques dans l'air. Autant dire que les augmentations de teneur en isotopes lourds varient d'un système évaporatoire à l'autre en intégrant ces différents facteurs de variations qui reflètent les conditions climatiques locales. Dans la pratique, l'enrichissement exprimé par rapport à la composition isotopique initiale au début du processus est de quelques unités $\delta$ en oxygène 18 (ou quelques dizaines d'unités $\delta$ en deuterium) sous les climats tempérés, pour atteindre 30 à 40 $\delta^{18} \mathrm{O}$ et $150 \delta^{2} \mathrm{H}$ dans les sebkhas sahariennes (fig. 6) exposées aux conditions les plus extrèmes d'aridité (Fontes et Gonfiantini, 1967, op. cit.).

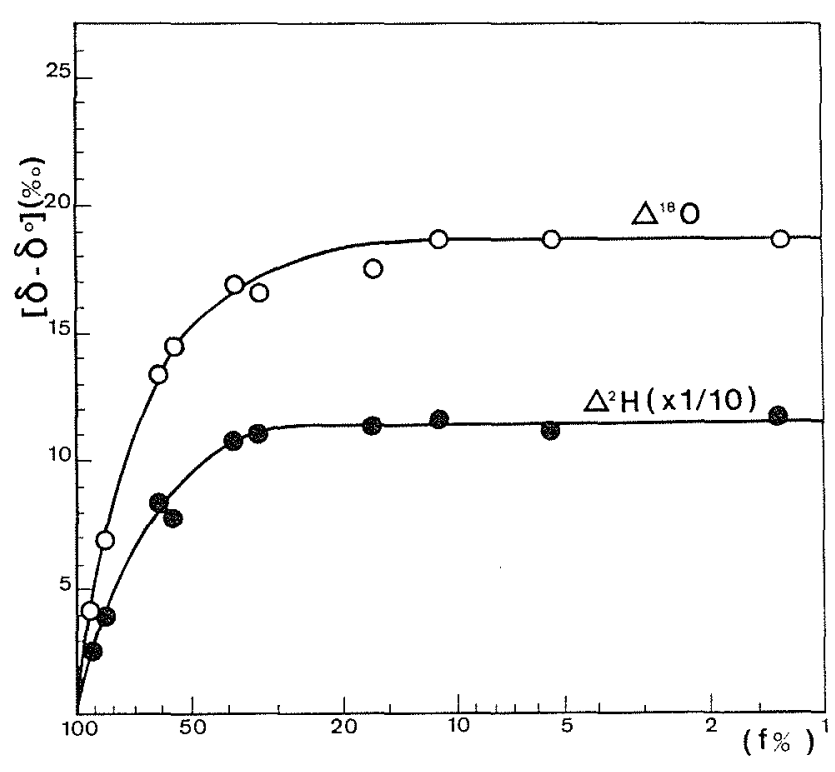

5/ Effet isotopique à l'évaporation : régions humides.

Evolution de la composition isotopique d'une eau en état d'évaporation avec enrichissement en isotopes lourds limité par l'échange avec la vapeur atmosphérique. La composition isotopique de l'eau tend vers une valeur constante, dite d'état stationnaire isotopique, alors que la fraction restante $f$ de liquide continue à dim:nuer. Expérience conduite en Californie avec une humidité relative élevée ( $h$ voisin de $80 \%)$. Simplifié, Craig et al., (1963).

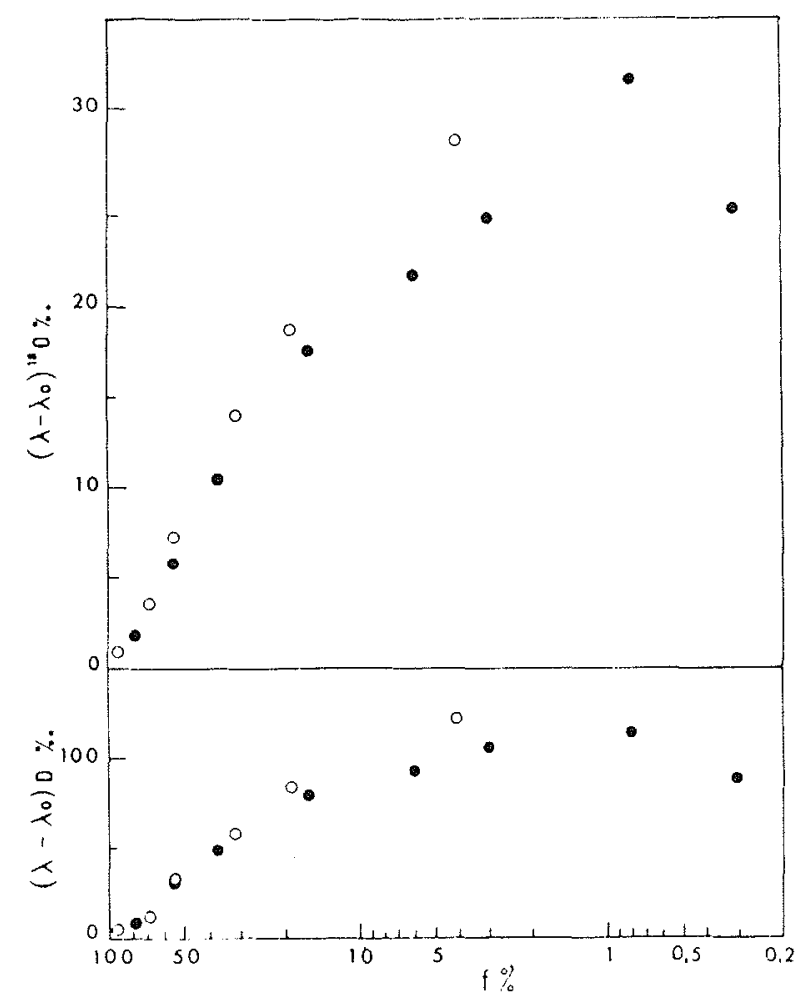

6/ Effet isotopique à l'évaporation : régions arides.

Les bassins du Nord-Ouest saharien, Lac du Guelb el Melah et Guelta de Gara Diba montrent un enrichissement isotopique intense au cours de leur évolution évaporatoire.

La fraction restante $f$ est calculée en admettant que le sodium (jusqu'à saturation en halite) puis le potassium sont conservatifs.

$$
\lambda=\ln (1+\delta 1)=\delta
$$

Cercles ouverts : Guelta ; cerctes pleins : Lac du Guelb el Melah. 
Les eaux de surface qui pénètrent dans le sol sont rapidement soustraites au phénomène d'évaporation libre et à l'enrichissement isotopique qui s'ensuit. Cependant, sous des climats arides comme celui du Nord du lac Tchad, il semble que l'évaporation puisse entrainer un effet isotopique jusque vers $3 \mathrm{~m}$ de profondeur (Bradley et al. en prép.). L'évapotranspiration qui affecte les eaux de la nappe à son toit par l'intermédiaire de la frange d'alimentation des poils radiculaires absorbants, semble devoir s'opérer sans enrichissement isotopique. Les végétaux se trouvent enrichis en isotopes lourds (Gonfiantini et al. 1965, Lesaint et al, 1974) par évapotranspiration par rapport à l'eau d'alimentation. Mais lorsque l'enrichissement est tel que la vapeur transpirée par les feuilles atteint la composition isotopique de l'eau de la nappe, le système est alors en régime permanent. Ce raisonnement qualitatif est facilement étayé par un traitement théorique en considérant que le végétal est un bassin évaporitique à niveau maintenu pratiquement sans échange avec la vapeur atmosphérique (Fontes, 1974).

\subsubsection{Traçage par réactions d'échange.}

D'autres causes possibles de variations de la composition isotopique des eaux dans les terrains aux basses températures ont été envisagées par certains auteurs. Elles concernent des effets assimilables aux phénomènes d'ultrafiltration, effets mal connus et de toutes façons relativement limités.

Aux fortes températures comme celles des champs géothermiques, l'eau entre en réaction avec les minéraux de l'encaissant. Ainsi on peut bien souvent relier l'échauffement d'une eau à sa charge en silice ou aux teneurs relatives en $\mathrm{Na}^{+}, \mathrm{K}^{+}$, $\mathrm{Ca}^{2+}$. De la même façon les teneurs en oxygène 18 des eaux ainsi réchauffées vont s'élever. En effet, silicates et carbonates sont très généralement plus riches en ${ }^{18} \mathrm{O}$ que les eaux. A hautes températures, le facteur de fractionnement diminuant, les teneurs en ${ }^{18} \mathrm{O}$ des eaux surchauffées vont tendre vers les teneurs de la roche. En revanche, les roches étant pratiquement dépourvues de minéraux hydrogénés, à l'exception des radicaux hydroxyles des micas et des amphiboles, il est normal qu'aucun effet particulier ne vienne perturber les teneurs en deuterium (fig. 7). Les eaux des circuits géothermiques vont être caractérisées par une augmentation des teneurs en ${ }^{18} \mathrm{O}$ sans modification des teneurs en deuterium (Craig, 1963, 1966 ; Gonfiantini et al. 1973)

Un autre type de réaction d'échange susceptible de modifier les teneurs isotopiques, est celle qui met en jeu un composé gazeux et l'eau.

Par exemple Ia réaction

$$
\mathrm{C}^{16} \mathrm{O}_{2}+\mathrm{H}_{2}^{18} \mathrm{O} \rightleftharpoons \mathrm{C}^{38} \mathrm{O}_{2}+\mathrm{H}_{2}^{16} \mathrm{O}
$$

engendre un enrichissement du $\mathrm{CO}_{2}$ en ${ }^{18} \mathrm{O}$ (de l'ordre de $40 \%$ à l'équilibre à $25^{\circ} \mathrm{C}$ ). Les eaux chaudes qui se sont trouvées au contact de grandes quantités de $\mathrm{CO}_{2}$ tendent à s'appauvrir en oxygène 18. C'est le cas par exemple de certaines eaux incrustantes (Ferrara et al., 1965).

L'hydrogène participe à des réactions d'échange affectées de fortes ségrégations isotopiques. Ainsi le contact avec de l'acide sulfhydrique $\left(\mathrm{H}_{2} \mathrm{~S}\right)$ entraine un enrichissement énorme de l'eau en deuterium (Nief et Botter, 1960).

\subsubsection{Relations ${ }^{18} \mathrm{O}-{ }^{2} \mathrm{H}$ dans les eaux naturelles.}

Tous les raisonnements qualitatifs qui précèdent s'appliquent aussi bien à l'oxygène 18 qu'au deuterium. Par exemple

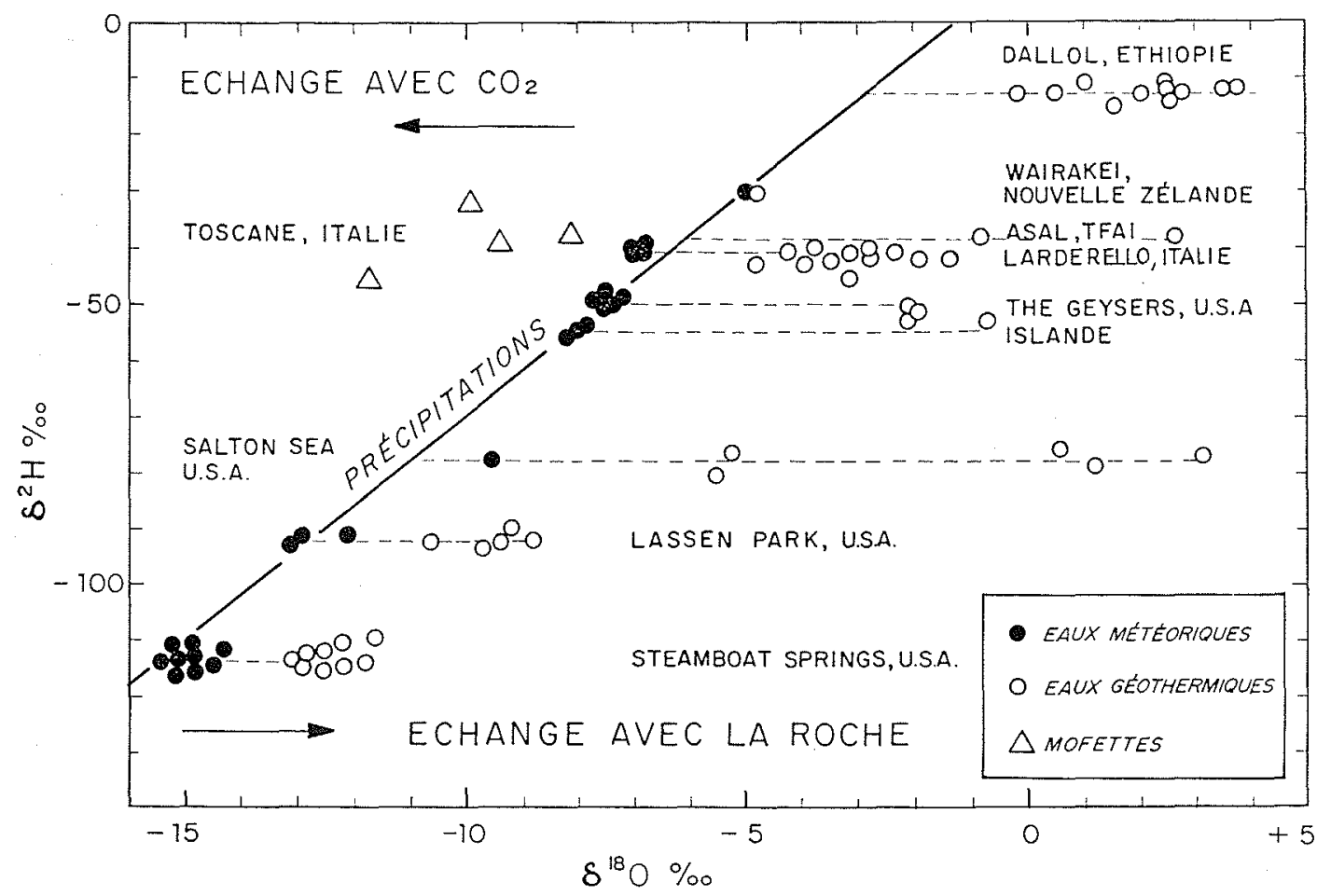

7/ Traçage isotopique par échange géothermique : principaux sites actuellement recensés.

Aux fortes températures des circuits géothermiques les eaux s'enrichissent en oxygène 18 par échange avec les roches encaissantes qui ont des teneurs en ${ }^{18} \mathrm{O}$ généralement plus fortes que celles des eaux. L'intersection d'une horizontale avec la droite des eaux météoriques représente les teneurs en ${ }^{18} \mathrm{O}$ et ${ }^{2} \mathrm{H}$ des eaux d'infiltration avant les réactions d'échange et permet de discuter de leur origine. Les eaux des mofettes très riches en $\mathrm{CO}_{2}$ subissent un échange isotopique qui conduit à un enrichissement du $\mathrm{CO}_{2}$ en ${ }^{18} \mathrm{O}$. Les eaux se trouvent donc appauvries par effet de bilan. 
lors des épisodes de précipitation dans la nature les teneuirs en ${ }^{18} \mathrm{O}$ et en ${ }^{2} \mathrm{H}$ sont, de façon très satisfaisante, respectivement corrélées linéairement à la température de condensation. Il est donc normal que les teneurs en isotopes lourds des eaux de précipitations soient codépendantes. Il est maintenant bien établi que les précipitations qui n'ont pas subi d'évaporation obéissent à la relation :

$$
\delta^{2} \mathrm{H}=8 \delta^{18} \mathrm{O}+d
$$

La bonne constance de la pente de 8 au cours de tous les phénomènes de précipitations est, schématiquement, due au fait que la condensation est toujours un phénomène d'équilibre puisqu'il intervient à saturation.

La quantité $d$, désignée sous le nom d'excès de deuterium, est dans la plupart des cas voisine de +10 , en particulier (cf $\S 3.2 .1 .2$.) pour les stations soumises à un régime de perturbations océaniques simples (H. Craig. 1961 b) : (fig. 8).

Lorsqu'une proportion notable de la vapeur condensante provient d'une mer fermée ou de l'évaporation continentale elle-mène, le paramètre $d$ varie. Par exemple pour le bassin oriental de la Méditerranée les compositions isotopiques des précipitations obéissent à la relation :

$$
\delta^{2} \mathrm{H}=8 \delta^{18} \mathrm{O}+22(\mathrm{Nir}, 1967)
$$

A l'évaporation les teneurs en ${ }^{18} \mathrm{O}$ et ${ }^{2} \mathrm{H}$ du liquide restent corrélées de façon linéaire pour un bassin donné. D une masse d'eau en état d'évaporation à l'autre, les pentes varient et traduisent en cela le fait que l'évaporation est pratiquement toujours un phénomène hors d'équilibre puisqu'il intervient en atmosphère dessaturée. Bien que variables, les pentes des droites qui traduisent la corrélation des teneurs en ${ }^{18} \mathrm{O}$ et ${ }^{2} \mathrm{H}$ de la phase liquide restante lors de l'évaporation, présentent cependant un caractère commun : elles sont toutes inférieures à la pente unique de 8 qui reflète le phénoméne d'équilibre à la condensation (fig. 9). Les nombreux résultats disponibles montrent que les droites d'évaporation ont généralement des pentes comprises entre 3 et 5 (voir à ce sujet Fontes, 1974, op. cit). A noter que les eaux des végétaux donnent lieu au même phénomène avec des pentes voisines de 3 .

\subsubsection{Le tritium isotope radioactif de l'hydrogène.}

En toute rigueur le tritium obéit aux lois du fractionnement telles qu'elles ont été énoncées pour les isotopes stables. Remarquons tout de suite que les effets isotopiques qui peuvent en découler ne peuvent être que de quelques dizaines de pour cent relatifs sur le rapport ${ }^{3} \mathrm{H} /{ }^{1} \mathrm{H}$. Bien qu'importantes ces causes de variations restent relativement faibles devant les autres effets qui affectent les teneurs en tritium et ne sont généralement pas prises en compte dans les études qui mettent en jeu cet isotope. On comparera directement les teneurs des précipitations à celles des eaux de surface et des nappes.

\subsubsection{Le tritium naturel.}

Fils des premiers noyaux de l'azote 14 de l'air soumis aux chocs de la composante neutronique induite par les protons du rayonnement cosmique, le tritium est engendré en haute atmosphère au taux moyen de 0,25 atomes $/ \mathrm{cm}^{2} / \mathrm{s}^{-1}$. (Lal et Suess, 1968). Après oxydation il est distribué à la surface du sol par les précipitations. La déviation des particules chargées du rayonnement cosmique par le champ magnétique terrestre étant décroissante avec la latitude, il est normal que des quantités majeures de tritium soient produites dans les régions de haute latitude.

Par ailleurs, dans les zones basses de l'atmosphère les masses de vapeurs subissent des réactions d'échanges à la surface des eaux libres. Les eaux continentales qui ont une origine continentale récente contiennent encore du tritium. En revanche les masses marines qui correspondent à des temps de séjour longs de plusieurs siècles à plusieurs millénaires sont évidemment très pauvres en cet isotope à mort assez précoce. L'échange avec les eaux océaniques appauvrit la vapeur atmos-

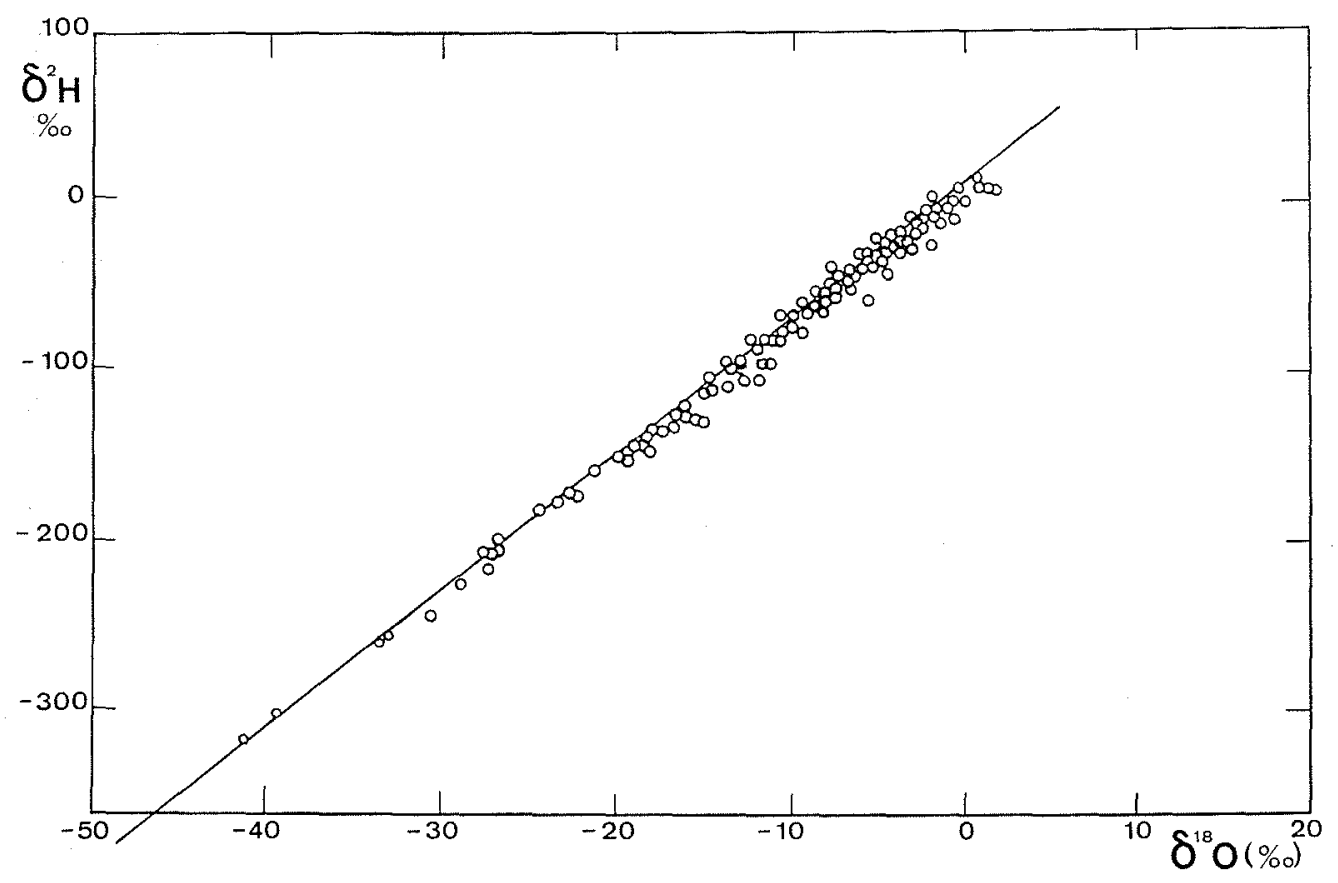

8/ Oxygène 18 et deutérium dans les précipitations.

A l'échelle mondiale, les teneurs en isotopes stables des précipitations sont bien corrélées au long d'une droite d'équation

$$
\delta^{2} H=8 \delta 180+10
$$

Simplifiè, d’après H. Craig (1961b). 


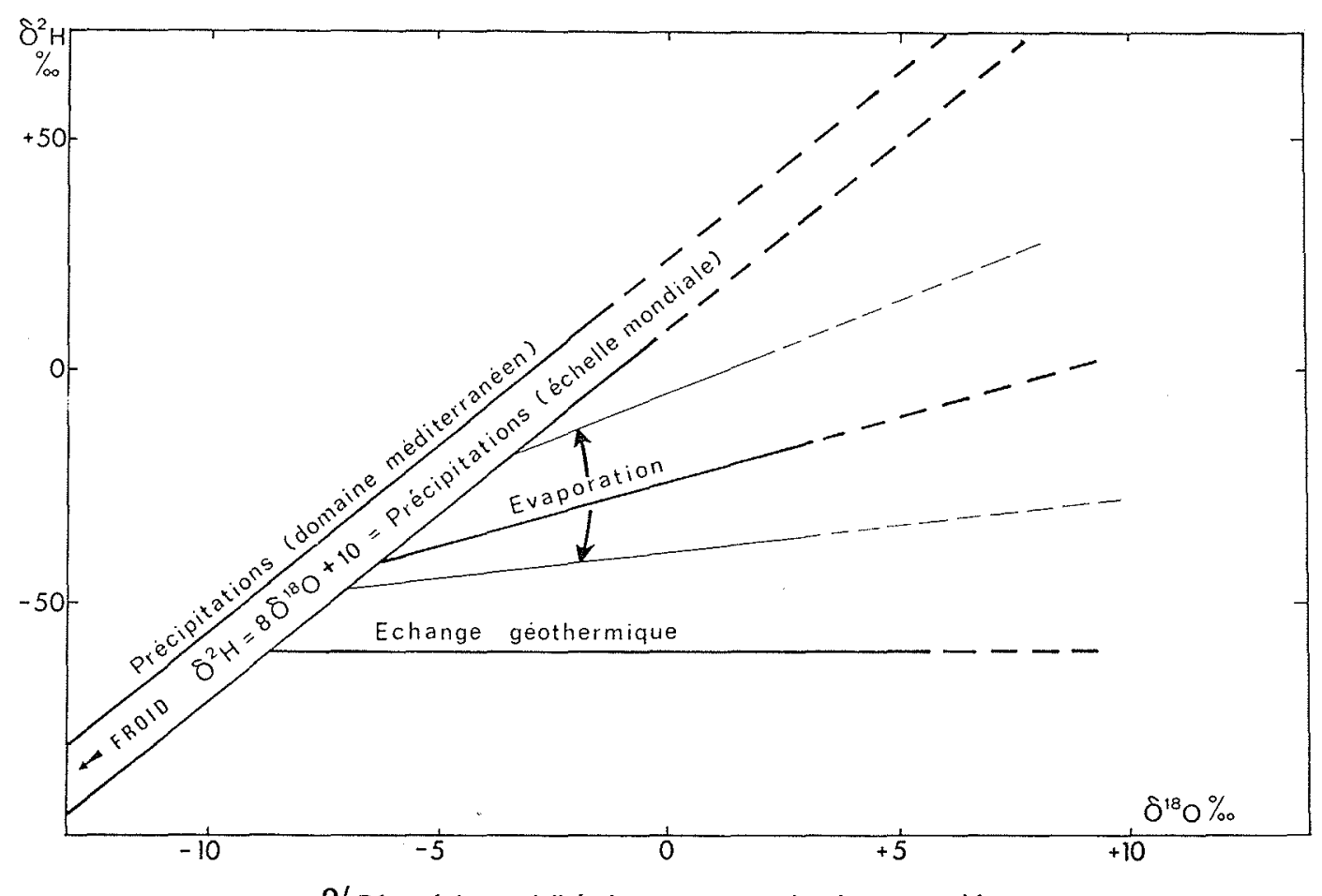

$9 /$ Résumé des modalités du traçage naturel en isotopes stables.

Les précipitations montrent une corrélation linéaire de pente 8 , caractéristique du phénomène de condensation, qui se produit toujours à saturation c'est-à-dire à l'équilibre. L'ordonnée à l'origine peut varier d'une région à l'autre en fonction de l'origine de la vapeur qui condense. La vapeur océanique (cas le plus fréquent) donne des précipitations qui ont un excés de deutérium de +10 . Les pluies du domaine méditerranéen oriental ont un excès de deutérium de +22.

L'évaporation zonduit également à des corrélations linéaires pour les teneurs en ${ }^{2} \mathrm{H}$ et ${ }^{18} \mathrm{O}$ des fractions liquides restantes. A la différence des précipitations qui obéissent à uné pente de 8 (pente d'équilibre), les eaux évaporées montrent des pentes variables qui traduisent le fait que chaque étendue d'eau en état d'évaporation obéit à sa cinétique propre qui dépend des paramètres climatiques locaux. De plus, les pentes des droites d'évaporation sont plus faibles que celle de la droite de condensation et généralement comprise entre 3 et 5 . Une eau évaporèe se repérera donc facilement en s'inscrivant sous la droite des précipitations.

L'échange géothermique n'affecte que les teneurs en ${ }^{18} \mathrm{O}$ qui se trouvent déplacées sur une horizontale.

phérique en tritium. D'autre part, et surtout, la contribution croissante de vapeurs marines au fur et à mesure de la migration des masses de vapeur au dessus des étendues océaniques dilue de plus en plus le tritium ambiant.

De même, les précipitations collectées en altitude ont plus de probabilité d'être assez largement tritiées que celles qui ont traversé la totalité des basses couches de l'atmosphère où les mćlanges avec la vapeur appauvrie en ${ }^{3} \mathrm{H}$ sont plus efficaces.

En principe, c'est donc au coeur des continents, aux hautes latitudes et altitudes que les teneurs en tritium sont les plus fortes. Dans la pratique seul l'effet de continentalité est vraiment important à l'échelle des valeurs moyennes pondérées, mensuelles ou annuelles (I.A.E.A., 1969, 1970, 1971, 1973). Conformément aux lois de variations définies ci-dessus les teneurs en tritium naturel des précipitations sont comprises entre 5 et 20 unités tritium (UT).

\subsubsection{Le tritium artificiel.}

Il est produit en fortes quantités lors des essais de bombes thermonucléaires et son apparition remonte donc aux premiers tests de 1954. Lors d'une expérience aérienne, le tritium se trouve largement injecté dans la stratosphère au delà de la zone écran de la tropopause. Là, soumis aux circulations laminaires zonales de cette enveloppe de l'atmosphère, il gravite à la latitude d'injection qui restera donc une zone préférentielle de retombées. Les retombées se produisent comme pour le tritium naturel sous forme humide, après oxydation et selon les mèmes mécanismes. Mais les teneurs artificielles ont été particulièrement élevées à certaines périodes et ont parfois dépassé de plusieurs ordres de grandeur les teneurs naturelles, autorisant ainsi des mesures plus faciles et plus précises (fig. 10). Les différents mécanismes qui favorisent la translation du tritium de la stratosphère vers la troposphère ont pu ètre ainsi précisés (Thatcher et Payne, 1965 ; Suess 1969 ; Olive, 1970).

La diffusion à travers la barrière tropopausique est un mécanisme qui joue probablement en permanence.

L'ensemencement direct des nuages convectifs à fort développement vertical est possible lorsque les têtes de ces cumulonimbus atteignent ou dépassent même la tropopause. Ce phénomène est plus facile vers les moyemes et hautes latitudes puisque la tropopause s'abaisse en moyenne de $12000 \mathrm{~m}$ à l'équateur jusque vers $6000 \mathrm{~m}$ aux póles.

Le délestage direct semble cependant le mécanisme le plus efficace de retombée du tritium. Il intervient à la faveur des discontinuités de la tropopause dont on ne sait si elles sont de véritables déchirures ou des extrusions dirigées vers le bas qui se trouvent découvertes à la fin du printemps. A cette époque, les tropopauses polaires et équatoriales ont tendance à migrer en sens inverses découvrant ainsi la zone par où se déversent les produits jusque là maintenus en sustentation stratosphérique. Par la suite les deux tropopauses convergent à nouveau et se recouvrent, oblitérant ainsi la zone de délestage. Il en résulte schématiquement un pic dit "pic de printemps" qui s'inscrit à environ 3 fois la teneur moyenne pondérée de l'année, suivi par une vallée d'hiver qui est sensiblement inférieure de moitié à cette même moyenne.

Les variations saisonnières des teneurs en tritium des précipitations se superposent à l'évolution générale qui depuis 1954 


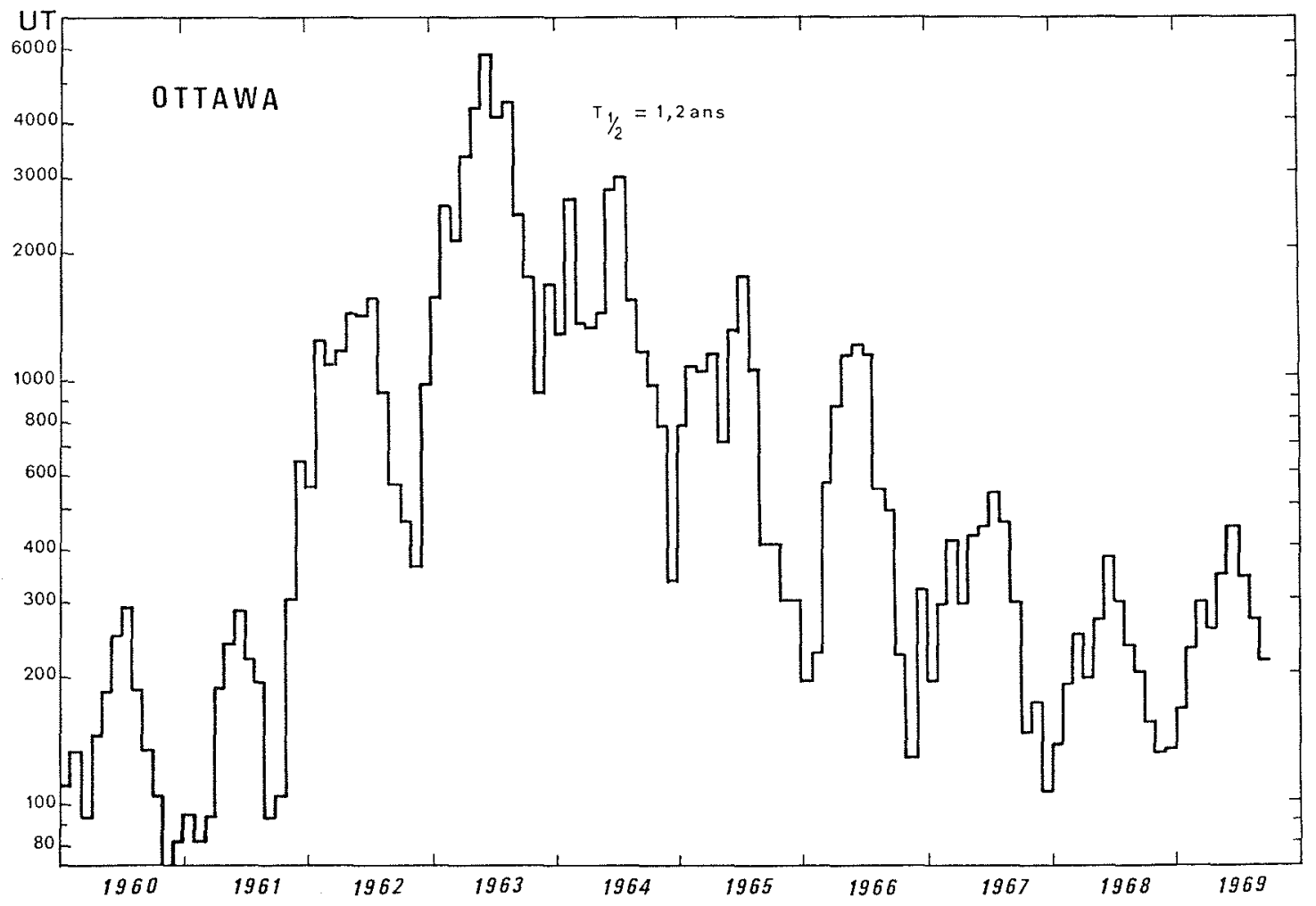

10/ Traçage des eaux naturelles en tritium.

Les variations de teneur en tritium des précipitations sont modutées par les essais thermonucléaires aériens. Après l'accord sur l'arrẽt de ces essais, les teneurs ont baissé de 1965 à 1969. Depuis 1970 l'activité des précipitations s'est stabilisée puis a commencé à augmenter à nouveau dans certaines régions sous l'effet des bouffées actives relâchées par les installations nucléaires.

La figure de retombée d'Ottawa qui correspond au plus long enregistrement disponible représente une archive de référence car les variations relatives sont sensiblement les mêmes dans tout I'hémisphère nord. Cette succession caractéristique de pics et de vallées représente la fonction entrée du tritium dans les nappes. Simplifié, d'après R. Brown (1970).

correspond au bilan injection-délestage du tritium artificiel bien connu dans l'hémisphère nord grâce aux enregistrements d'Ottawa qui remontent à 1953 (Brown, 1961, 1970).

Après une succession d'impulsions qui conduisent à des teneurs maximales au cours des printemps 1958-1959 les activités décroissent pour ne plus représenter que 2 à 3 fois le niveau naturel au cours de l'hiver 1960. Puis vient la période de la compétition aux essais d'engins toujours plus puissants. Elle culmine avec des retombées humides en tritium comprises entre 5000 et 10000 UT lors du "pic de printemps" de 1963 en Europe centrale et au centre du continent nord-américain. Par la suite avec l'accord sur l'arrêt des essais aériens, le réservoir stratophérique s'épuise rapidement jusqu'en 1968 avec un temps de demi-vidange de l'ordre de 1,2 ans. Depuis, la tendance se stabilise avec des fluctuations probablement imputables à des bouffées actives relachées par les réacteurs nucléaires, chaque année plus nombreux dans les régions industrialisées. A l'heure actuelle les teneurs moyennes en tritium des précipitations sur les régions tempérées de l'hémisphère nord s'inscrivent à environ 100 TU.

Dans l'hémisphère sud la tendance est complètement différente (fig. 11). En effet les circulations atmosphériques interhémisphères sont très faibles et le passage des masses d'air est donc lent. De plus ces circulations ne se produisent guère qu'au niveau des grandes étendues continentales, c'est-à-dire essentiellement en Afrique. Or, jusqu'aux essais de Polynésie l'hémisphère sud était resté exempt de tout apport intrinsèque. Si l'on ajoute que la surface marine australe, véritable piège à tritium, est relativement plus importante que celle des mers boréales, on concevra facilement que les teneurs en tritium

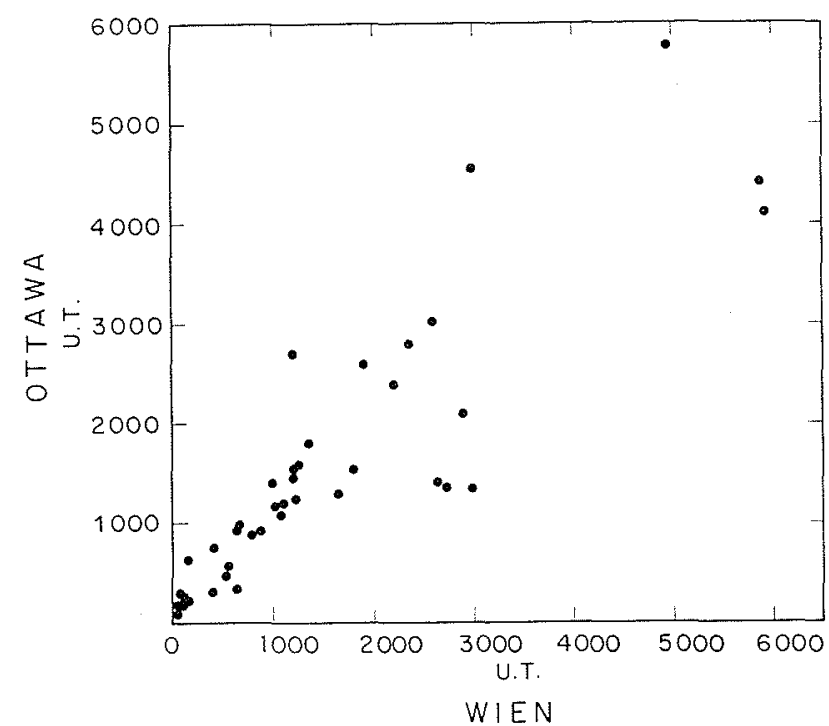

11/ Exemple de corrélation des teneurs en tritium entre deux stations du même hémisphère

Entre les stations de Vienne et d'Ottawa, la corrélation est suffisamment significative pour permettre la reconstitution de la fonction entrée à Vienne avant que ne débutent les mesures systématiques. Sources: R. Brown (1970) et I.A.E.A. (1969, 19701 .

soient restées faibles en particulier en Nouvelle Zélande et en Australie où elles n'ont point dépassé 40 à $50 \mathrm{UT}$, maximum relevé en 1965/1966 et 1966/1967 (Brown et Taylor, 1974 ; Allison et Hugues, 1974). Sur l'Afrique du Sud où l'apport 


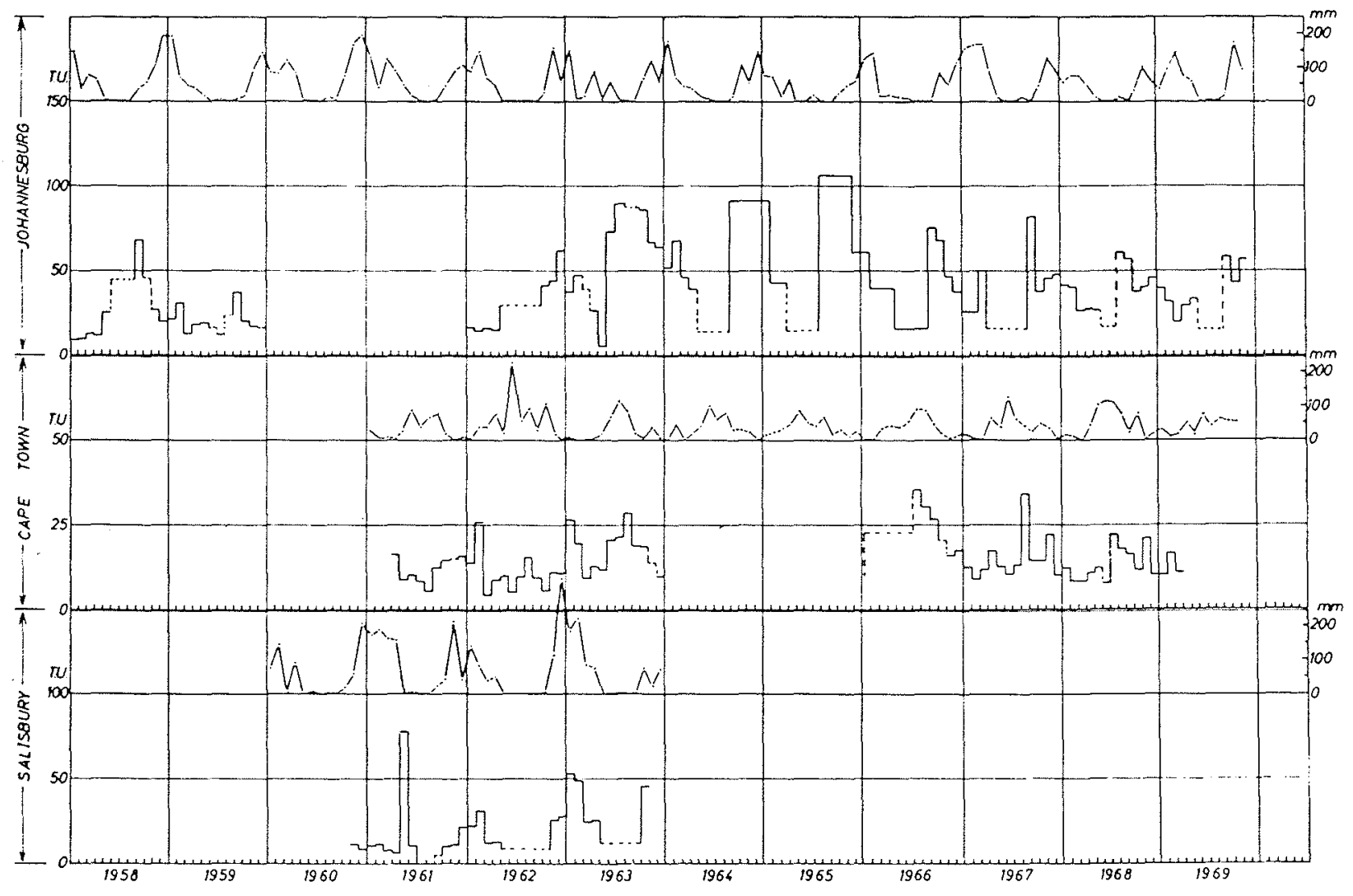

12/. Le tritium dans I'hémisphère sud (Afrique du Sud).

Les communications atmosphériques interhémisphères sont réduites et difficiles. Les injections d'origine thermonucléaires s'étant próférentiellement produites dans I'hémisphère boréal, il est normal de relever des teneurs beaucoup plus basses et légèrement déphasées dans

les précipitations d'Afrique du Sud. D'après Verhagen et al. , (1970).

d'air boréal est plus aisé, le maximum de 1965 apparaît encore, légèrement décalé par rapport à celui de l'hémisphère nord (1963-1964) et reste inférieur à 110 UT (Vogel et Van Dijken, 1972).

\subsubsection{Traçage du milieu en tritium.}

Dans la pratique, et pour ce qui regarde l'hémisphère nord, les facteurs qui tendent à provoquer des variations des retombées en tritium d'une région à l'autre se trouvent fortement contrariés par le "bon" brassage turbulent de la troposphère. Pour chaque grande zone climatique les teneurs en tritium des précipitations restent simplement proportionnelles entre elles d'une localité à l'autre, traduisant ainsi les effets de la continentalité. Cette intéressante propriété permet de reconstituer a posteriori les teneurs en tritium précipité à une station donnée, en se référant aux enregistrements plus complets d'une autre station. Etant donné que la collection de données continues d'Ottawa couvre la totalité de l'évolution des retombées en ${ }^{3} \mathrm{H}$ de 1953 à 1969 (Brown, 1970 op. cit). il a été possible de recaler de façon précise toutes les figures complètes locales de retombées de la zone tempérée au prix de quelques années de mesures destinées à établir les divers coefficients de proportionnalité avec Ottawa (fig. 12). La courbe de distribution des teneurs en tritium des précipitations en fonction du temps depuis 1953 représente la fonction entrée fondamentale de traçage en tritium. La comparaison des données de cette courbe avec les variations d'activité au cours du temps, d'un cours d'eau ou d'un point d'eau souterrain,permet de procéder aux mémes types de traitements que dans la pratique du traçage artificiel (fig. 13). La fonction entrée est connue, la fonction distribution des temps de séjour est déterminée, le traitement par déconvolution permet de reconstituer les paramètres

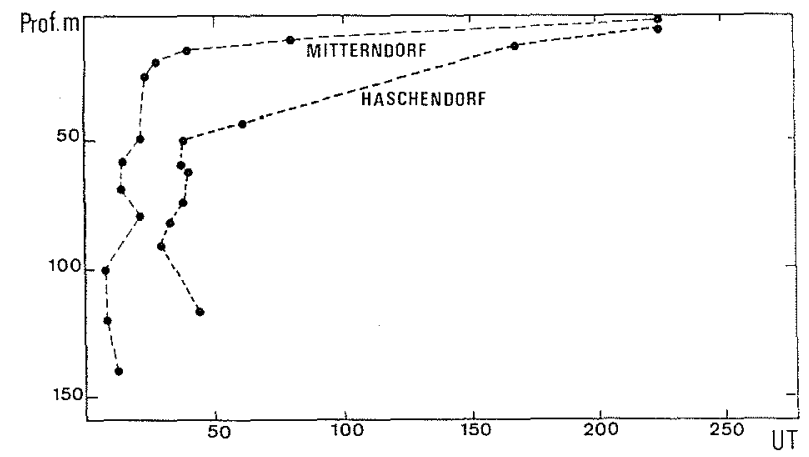

13/. Tritium indicateur des variations de perméabilité.

Les teneurs en tritium des eaux prélevées au cours de l'éxécution de deux forages en Autriche sont reportées en fonction de la profondeur. Les eaux sont récentes mais stratifiées en fonction des variations de perméabilité verticale des terrains. Simplifié, d'après Davis et al. (1967).

de dispersion du système (Nir, 1964 ; Margrita et al, 1970). D'autres approches sont également possibles comme celle qui consiste à établir des modèles de mélanges successifs où chaque année la même proportion d'eau récente se mêle à un mélange des eaux des années précédentes obtenu de la même façon et homogène (Münnich et Roether,1963; Davis et al. 1967). Plus simplement le tritium est souvent utilisé sur la base d'estimations empiriques qui permettent d'identifier les mélanges. Aussi pour la zone tempérée nord on peut admettre les critères suivants pour l'étude des eaux souterraines:

-0 à 5 UT la composante ancienne antérieure à 40 ans domine ;

- 5 à 40 UT le prélèvement représente un mélange entre de l'eau ancienne et des apports récents ; 
- >40 UT la composante contemporaine domine.

Encore faut-il bien voir qu'il ne s'agit là que de recettes destinées à guider les enquêtes et non de règles. Il est clair par exemple qu'une contamination d'une réserve souterraine vieille de plusieurs millénaires par $2 \%$ d'eau météorique de Juin 1963 conduira, compte tenu de la décroissance radioactive du tritium, à une teneur de 40 à 50 UT en 1976. Bien entendu la logique continuelle du système hydraulique rend hautement improbable une telle coincidence, mais il convient d'être prudent en particulier dans le cas des karsts où les décharges en tritium au moment des crues peuvent être le produit de mélanges discontinus (Margrita et al, 1970, op. cit).

Parfois encore le tritium est utilisé en tout ou rien pour diagnostiquer le passage des eaux de 1964 dans les systèmes qui sont censés fonctionner essentiellement par effet piston (Smith et al, 1970) : (fig. 14)

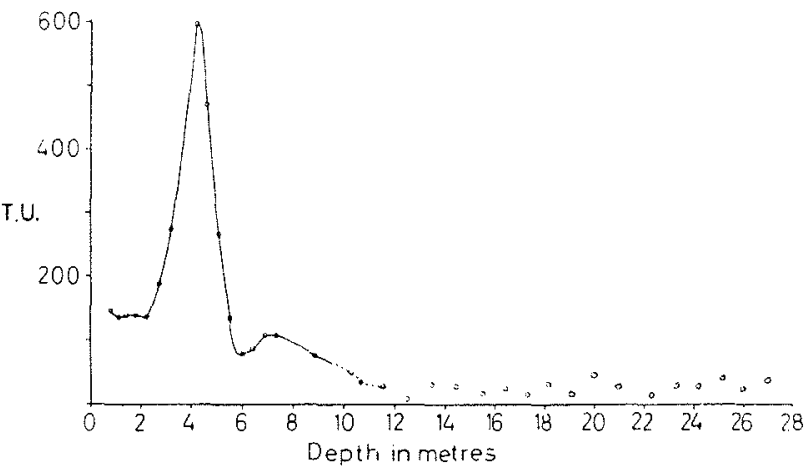

14/. Tritium indicateur de la stratification des eaux dans la zone non saturée.

Le pic de tritium attribué aux précipitations des années 1963-1964 (pic majeur) est retrouvé, pratiquement sans dispersion, dans un profil de craie à porosité d'interstice homogène ot forte (teneur en eau : $0,21 \mathrm{~g} / \mathrm{g}$ ).

Le pic qui précède est attribué au pic secondaire des années $1958-1959$ (cf. fig. A-10). Les recharges des années successives s'empilent à raison de $0,88 \mathrm{~m} / \mathrm{an}$, soit une recharge annuefle d'environ $330 \mathrm{~mm} / a n$ pour une porosité totale de $38 \%$. D'après Smith et al. (1970).

\subsection{ISOTOPES DU MILIEU EN SOLUTIONS : TRACEURS EXTRINSEQUES DE L'EAU.}

3.3.1. Isotopes du carbone dissous ${ }^{13} \mathrm{C},{ }^{14} \mathrm{C}$.

3.3.1.1. Production du ${ }^{14} \mathrm{C}$ et conditions d'emploi en marqueur de temps.

Comme le tritium, le radiocarbone ${ }^{14} \mathrm{C}$ se forme dans la haute atmosphère à partir des noyaux d'azote 14 .

$$
{ }^{14} \mathrm{~N} \stackrel{n, p}{\longrightarrow}{ }^{14} \mathrm{C}
$$

Après oxydation il est distribué dans le cycle général du carbone sous forme initiale de ${ }^{14} \mathrm{CO}_{2}$ mélé au $\mathrm{CO}_{2}$ atmosphérique. Le principe de l'utilisation du carbone 14 comme chronomètre est fondé sur l'estimation de la décroissance radioactive par unité de temps qui est proportionnelle au nombre de nuclides $N$ du milieu

$$
d N=N \lambda d t
$$

qui s'intègre en considérant l'état initial $\left(t=0, N=N_{0}\right)$ :

$$
N=N_{0} e^{-\lambda t}
$$

ou encore en introduisant la période radioactive ou temps de demi-vie $\left(t=\tau, N=\frac{N_{0}}{2}\right)$;

$$
t=\frac{\tau}{\ln 2} \ln \frac{N_{0}}{N_{t}}
$$

Dans le cas des marqueurs de temps généralement utilisés en géochimie on détermine $N_{o}$ avec la teneur en noyaux fils produits dans la réaction de décroissance et qui est égale à la différence $N_{0}-N_{1}$. lci le noyau fils du carbone est l'azote banal ${ }^{14} \mathrm{~N}$.

$$
{ }^{14} \mathrm{C} \rightarrow{ }^{14} \mathrm{~N}+\beta^{-}
$$

L'azote 14 radiogénique, dilué à l'infini par l'azote atmosphérique est évidemment inaccessible.

Pour déterminer le temps par la mesure de l'activité spécifique $A_{t}=N_{t} / g$ on doit donc estimer $A_{o}$.

L'hypothèse la plus simple consiste à admettre que $A_{o}$ reste constant au cours du temps et correspond à l'activité du $\mathrm{CO}_{2}$ de l'atmosphère. Cela suppose :

- un équilibre entre la production cosmique et la décroissance radioactive $\mathrm{du}{ }^{14} \mathrm{C}$

- une bonne homogéneisation du réservoir troposphérique

- un cycle du carbone 14 en régime permanent en chaque point

la connaissance des fractionnements isotopiques qui accompagnent les réactions du cycle du carbone

Toutes ces conditions liminaires nécessitent un examen critique délicat. Disons simplement que la comparaison des âges radiométriques avec les âges réels a pu être menée sur les 6 derniers millénaires avec des matériaux tirés des nécropoles et surtout par dendrochronologie, c'est-à-dire par comptage des anneaux de croissance de certains arbres (Pinus aristata du sud des Etats-Unis). Les résultats montrent un écart croissant avec le temps à partir du début de notre ère. En limitc d'étalonnage la différence est d'environ 10\% (Damon et al. 1966)

Pour le calcul du temps deux contraintes propres au milieu d'ćtude s'ajoutent à ces conditions :

- le système doit rester clos, c'est-à-dire exempt de toute contribution supplémentaire en carbone actif ou "mort" qui viendrait fausser l'effet de la décroissance radioactive du ${ }^{14} \mathrm{C}$

- l'activité initiale du système au moment de sa fermeture $A_{o}$ doit pouvoir être reliée à l'activité du $\mathrm{CO}_{2}$ de l'atmosphère au même moment.

La notion de système clos est assez facile à admettre dans le cas de restes végétaux massifs tels que les bois. Pour les carbonates ou les bicarbonates dissous dans les eaux souterraines, il ne peut y avoir de système clos qu'en nappe captive. Le problème de l'activité initiale est lié à l'origine, c'est-à-dire au chimisme du carbone dissous.

3.3.1.2. Pénétration du carbone dans les cycles hydrologiques, activité initiale du carbone dissous.

La pénétration du carbone dans l'hydrosphère suit donc deux voies différentes: (fig. 15)

-- équilibre $\mathrm{CO}_{2}-\mathrm{CO}_{3} \mathrm{H}_{2}$ à la surface des eaux libres :

- organo-synthèse via la phytosphère et l'assimilation chlorophylienne.

Après fermentation ou même seulement par respiration, la voie organique conduit elle-même à un dégagement de $\mathrm{CO}_{2}$ par décarboxylation. $\mathrm{Ce} \mathrm{CO}_{2}$ est alors disponible pour s'intégrer à la suite des équilibres de dissociation des bicarbonates et des carbonates de la même façon que dans la voie minérale. Cependant, la mise en solution du $\mathrm{CO}_{2}$, premier terme de la série d'équilibres, est proportionnelle à la pression partielle du $\mathrm{CO}_{2}$ gazeux (loi de Henry). 


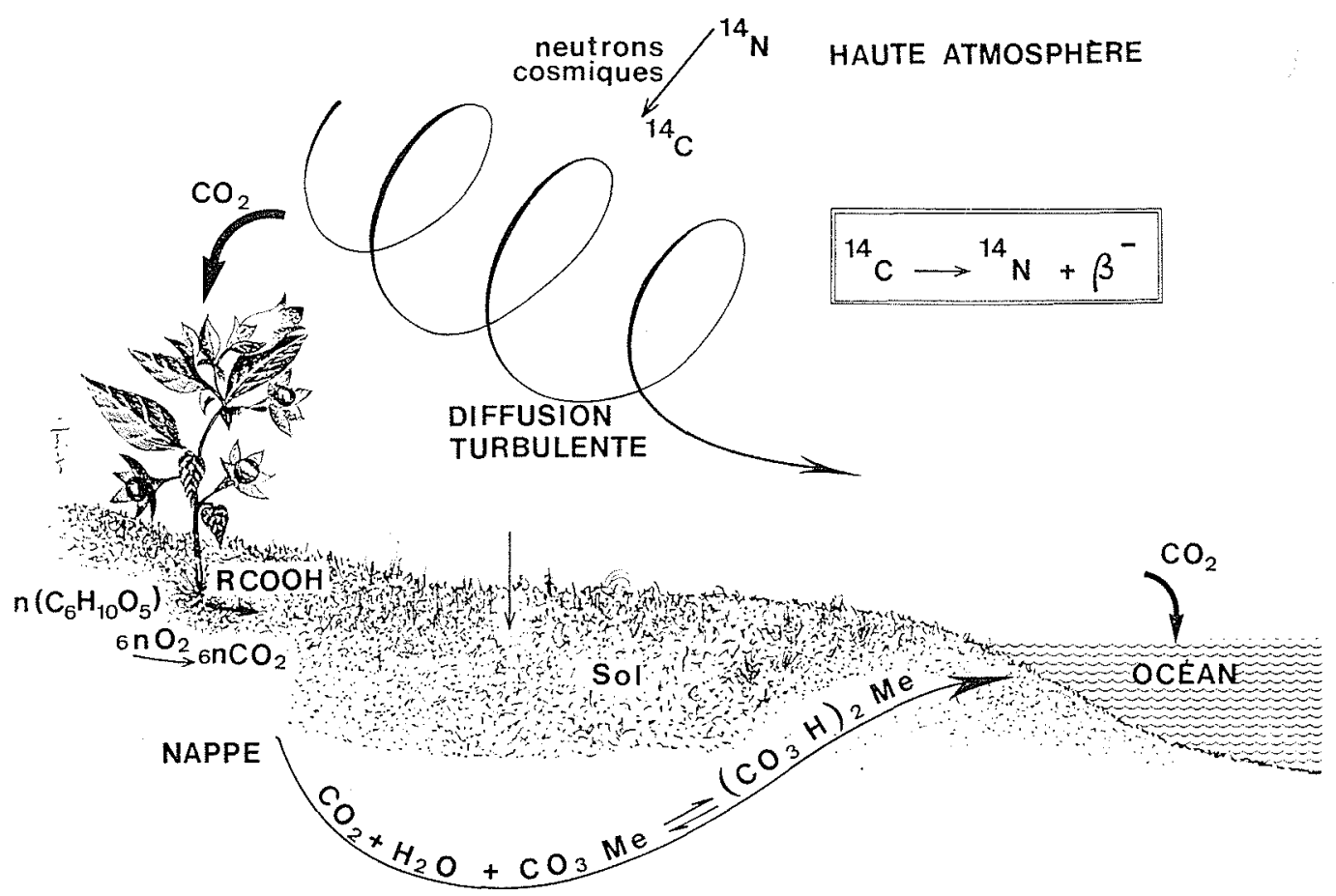

15/. Cycle du carbone 14 naturel dissous.

Après sa production par les rayons cosmiques, le carbone 14 est brassé dans la troposphère sous forme de gaz carbonique. II pénètre ensuite dans les autres portions du cycle selon la voie directe par réalisation des équilibres $\mathrm{CO}_{2}-\mathrm{CO}_{3} \mathrm{H}_{2}-\mathrm{CO}_{3} \mathrm{H}^{-}$à la surface des eaux libres (ou encore, pour mémoire, par infiltration directe des eaux chargées en $\mathrm{CO}_{2}$ dissous) ou bien, et c'est la voie préférentielle pour la partie continentale du cycle, le $\mathrm{CO}_{2}$ de l'atmosphère est assimilé par les végétaux chlorophylliens et se trouve ensuite cédé à nouveau à l'hydrosphère par oxydation ou décarboxylation de la matière organique.

L'eau agressive chargée en acide carbonique est ensuite saturée par des cations, le plus souvent alcalino-terreux, pour donner les bicarbonates qui circulent dans la zone noyée. La réaction de l'acide carbonique actif (= chargé en ${ }^{14} \mathrm{C}$ ) qui provient du sol sur le carbonate "mort" (= dépourvu de ${ }^{\mathrm{A}} \mathrm{C}$ ) peut se résumer à une dilution de l'activité du $\mathrm{CO}_{2}$ de l'atmosphère dans le carbone dissous. La dáter. mination des termes de cette dilution, c'est-à-dire de l'activité initiale du carbone dissous représente le problème crucial dans l'estimation des "âges" des eaux anciennes.

Sous l'effet de l'activité organique la pression partielle de $\mathrm{CO}_{2}$ dans les sols est d'environ deux ordres de grandeur supérieure à celle de l'atmosphère $\left(10^{-3,4} \mathrm{~atm}\right)$. Les sols constituent, de loin, le point de plus large passage du carbonc entre l'atmosphère et la partie continentale de l'hydrosphère.

Le $\mathrm{CO}_{2}$ qui se dissout dans l'eau des sols donne le départ à une chaine de réactions du type :

$$
\begin{aligned}
& \mathrm{CO}_{2} \text { (gaz) } \rightleftharpoons \mathrm{CO}_{2} \text { (liq.) } \\
& \mathrm{CO}_{2} \text { (liq.) }+\mathrm{H}_{2} \mathrm{O} \rightleftharpoons \mathrm{H}_{2} \mathrm{CO}_{3} \\
& \mathrm{H}_{2} \mathrm{CO}_{3} \rightleftharpoons \mathrm{HCO}_{3}^{-}+\mathrm{H}^{+} \\
& \mathrm{H}^{+}+\mathrm{CO}_{3} \mathrm{Me} \text { (sol.) } \rightleftharpoons \mathrm{HCO}_{3}^{-}+\mathrm{Me}^{2+}
\end{aligned}
$$

qui reviennent à la mise en solution d'un carbonate solide, lc plus souvent alcalino-terreux $(\mathrm{Me}=\mathrm{Ca}$ ou $\mathrm{Mg}$ ) sous l'action de l'eau agressive.

$$
\mathrm{CO}_{3} \mathrm{Me}+\mathrm{CO}_{2}+\mathrm{H}_{2} \mathrm{O} \rightleftharpoons\left(\mathrm{CO}_{3} \mathrm{H}\right)_{2} \mathrm{Me}
$$

On devrait donc considérer l'activité du carbone dissous sous forme de bicarbonate comme le résultat d'un mélange équiatomique des deux espèces de carbone : le $\mathrm{CO}_{2}$ portcur de ${ }^{14} \mathrm{C}$ et le carbonate solide généralement dépourvu de radiocarbone. Avec $100 \%$ de carbone moderne dans le $\mathrm{CO}_{2}$ d'origine biologique et $0 \%$ dans le carbone minéral, on obtient facilement une activité initiale théorique de $50 \%$ pour le bicarbonate dissous.

Dans la réalité plusieurs mécanismes interviennent pour modifier ce trop simple schéma réactionnel : le carbone dissous comprend en général la coexistence d'au moins deux formes chimiques $\left(\mathrm{CO}_{2}\right.$ dissous- bicarbonate ou bicarbonate-carbonate);

- la relation phase liquide-phase solide ne correspond pas toujours à des réactions d'équilibre, et donc à la stoechiométrie, puisque le calcul montre que les eaux souterraines sont très souvent sursaturées vis-à-vis des carbonates;

-- le carbone en phase liquide est susceptible d'échanges isotopiques, soit avec un excès de $\mathrm{CO}_{2}$ soit avec les carbonates solides de l'aquifère (Thilo et Münnich, 1970)

\subsubsection{Différents modèles d'estimation de l'activité initiale.}

Des approches différentes de l'activité initiale ont été proposées. Vogel $(1967,1970)$ réalise une étude statistique de la teneur en ${ }^{14} \mathrm{C}$ du carbone dissous dans l'eau des sols d'Allemagne. A l'issue de plus de 100 mesures, il propose la relation :

$$
A_{o}=85 \pm 5 \% \text { de carbone moderne. }
$$

La critique de ce modèle réside essentiellement dans le fait qu'il ne prend pas en compte les modifications de la composition chimique et isotopique qui interviennent après le passage dans les sols.

Tamers $(1966,1970,1975)$ propose une correction fondée sur les teneurs molaires respectives en $\mathrm{CO}_{2}$ dissous $a$ et en bicarbonate $b$. En attribuant l'activité au $\mathrm{CO}_{2}$ et à la moitié des bicarbonates, il vient :

$$
A_{o}=\frac{a+b / 2}{a+b} \% \text { carbone moderne }
$$


Ce modèle purement chimique méconnaît toute possibilité d'échange isotopique.

Pearson $(1970,1972,1974)$ adopte une méthode fondée sur la large discrimination isotopique qui se produit dans le cycle du carbone 13, isotope stable du carbone. Les végétaux lors de la biosynthèse utilisent avec préférence le carbone banal ${ }^{12} \mathrm{C}$. Cette règle de fonctionnement, très générale de l'activité enzymatique, conduit à un appauvrissement en ${ }^{13} \mathrm{C}$ de la matière organique, et donc du $\mathrm{CO}_{2}$ des sols par rapport au $\mathrm{CO}_{2}$ du réservoir atmosphérique. En revanche, les carbonates sédimentaires sont enrichis en ${ }^{13} \mathrm{C}$ par rapport à ce même $\mathrm{CO}_{2}$. Les carbonates marins ont une composition isotopique voisine de zéro, ce qui est normal puisque l'étalon de l'échelle destinée à repérer les teneurs en ${ }^{13} \mathrm{C}$ est un carbonate de rostre de Belemnite (P.D.B.I.). L'écart le plus généralement relevé entre carbonate marin et $\mathrm{CO}_{2}$ du sol est environ de $20 \%$ en ${ }^{13} \mathrm{C}$. En considérant que toutes les réactions se résument à des mélanges, il vient :

$$
\begin{aligned}
A_{0}=\frac{\delta_{\text {carbone tot, dissous }}-}{} & \delta_{\text {carbonate solide }} \\
\delta_{\mathrm{CO}_{2} \text { sol }}- & \delta_{\text {carbonate solide }} \\
& \simeq \frac{\delta_{\text {carb. total dissous }} \% \text { carb. moderne. }}{-25}
\end{aligned}
$$

Ce traitement simple conduit souvent à des valeurs cohérentes de $A_{o}$ mais il se révèle trop rigide en ce qui concerne les teneurs limites en ${ }^{13} \mathrm{C}$ du $\mathrm{CO}_{2}$ des sols d'une part, du carbonate de l'aquifère d'autre part.

Mook (1972) allie les traitements chimiques et isotopiques précédents et introduit les différents facteurs de fractionnement. L'expression du bilan chimique et isotopique à l'issue des réactions s'écrit alors:

$$
\begin{aligned}
A_{0}= & \frac{1}{a+b}\left\{a A_{a o}+b A_{b o}\right. \\
& \left.+\left(A_{b e}-A_{b o}\right) \frac{(a+b) \delta_{\Sigma}-a \delta_{c o}-b \delta_{b o}}{\delta_{b e}-\delta_{b o}}\right\} \% \text { carb. mod. }
\end{aligned}
$$

où $a$ et $b$ sont les concentrations en $\mathrm{CO}_{2}$ dissous et en bicarbonates, avec $\Sigma=a+b$, les indices $O$ et $e$ désignent respectivement les états initiaux et finaux après équilibration isotopique, $A$ les activités du ${ }^{14} \mathrm{C}, \delta$ les teneurs en ${ }^{13} \mathrm{C}$.

$\mathrm{Ce}$ modèle est beaucoup plus raffiné que les précédents mais se révèle très sensible aux variations de certains paramètres, en particulier $\delta_{a o}$.

Fontes et Garnier (1976) proposent une approche fondée sur le bilan chimique des éléments liés aux carbonates solides mobilisés (alcalino-terreux et éventuellement carbonates alcalins). La concentration en carbone mort $C_{M}$ est alors donnée par :

$$
m C_{M} \simeq m \mathrm{Ca}^{2+}+m \mathrm{Mg}^{2+}+m \frac{\mathrm{Na}+\mathrm{K}-\mathrm{Cl}}{2}-m \mathrm{SO}_{4}^{2-}
$$

L'échange isotopique du carbone dissous est traité comme un mélange partiel, d'une fraction ayant conservé sa composition isotopique et son activité initiales, avec une fraction totalement équilibrée avec l'encaissant carbonaté ou le $\mathrm{CO}_{2}$ en excès. L'équation finale est :

$$
\begin{aligned}
& A_{o}=\frac{1}{C_{T}}\left\{\left(C_{T}-C_{M}\right) A_{a o}+C_{M} A_{M}+\left(A_{a o}+\frac{2 \epsilon}{10}-A_{M}\right)\right. \\
& \left.\frac{\delta_{T} C_{T}-\delta_{M} C_{M}-\delta_{a o}\left(C_{T}-C_{M}\right)}{\left(\delta_{a o}+\epsilon\right) \cdot \delta_{M}}\right\} \% \text { de carbone moderne. }
\end{aligned}
$$

où $C$ désigne les concentrations, $A$ les activités ${ }^{14} \mathrm{C}$ et $\delta$ les compositions isotopiques; les symboles $T, M$ et ao se rapportent respectivement au carbone total dissous, au carbone mort mobilisé et au $\mathrm{CO}_{2}$ du sol ; $\varepsilon$ est pris comme le facteur de fractionnement entre le $\mathrm{CO}_{2}$ gazeux et le carbonate solide ( $\epsilon$ positif).

Ce modèle semble plus cohérent que les approches précédentes mais reste encore imparfait vis-à-vis en particulier d'apports en $\mathrm{CO}_{2}$ dans l'aquifère lui-mème par exemple par oxydation de la matière organique ou par exhalaisons profondes.

\subsubsection{Recours aux isotopes du carbone en hydrologie.}

A l'issue de la brève revue critique des divers modèles disponibles dans la littérature pour estimer les conditions aux limites de l'entrée du radiocarbone dans les nappes captives, il apparait que le problème n'est pas encore définitivement réglé.

Les études de terrain qui apportent suffisamment d'éléments pour pouvoir comparer les différentes approches en présence ne sont pas assez nombreuses. En effet, il est clair que toutes les études d'estimation du temps de transit souterrain des eaux selon un parcours souterrain défini par les points de collecte exige non seulement la précipitation totale du carbone dissous dans les eaux sous forme de carbonate de baryum en milieu alcalin, mais aussi la mesure in situ du $\mathrm{pH}$, de la température, du potentiel d'oxydo-réduction, de l'alcalinité, ainsi que l'analyse complète au laboratoire des ions majeurs avec un soin tout particulier pour les alcalinoterreux. Tout ceci requiert le déplacement sur le terrain d'un véritable laboratoire capable de réaliser tous ces traitements et de filtrer un témoin sur membrane colloidale avant de le fixer à l'acide chlorhydrique suprapur afin de stabiliser les teneurs en ions calcium et magnésium avant les analyses en laboratoire des ions majeurs dont le bilan sera complété sur un témoin non acidifié.

En certains cas les auteurs n'ont point cherché à reconstituer une valeur pour l'activité initiale et se sont limités à la simple hypothèse que cette activité est restée constante tout au long de la période d'infiltration de la nappe captive (Evin et Vuillaume, 1970) (Fig. 16). En ce cas $A_{o}$ disparaît de la relation qui donne la différence de temps de parcours entre deux points $A$ et $B$ de l'aquifère au long d'une direction d'écoulement :

$$
t_{B}-t_{A}=\frac{\tau}{\ln 2} \ln \frac{A_{B}}{A_{A}}
$$

Bien entendu cette approche ne tient pas compte des variations des nombreux paramètres du chimisme et de la répartition isotopique du carbone sous l'effet en particulier des variations climatiques dans les périodes, parfois fort longues, et fort différentes, sur lesquelles s'est étalée la recharge d'une nappe captive. Les modifications de composition chimique en cours de cheminement de l'eau ne sont pas considérées dans cette hypothèse très simple qui ne peut représenter qu'une large approximation. Une tentative de correction en a été proposée en considérant que l'augmentation de l'alcalinité en cours de trajet souterrain était due à une dilution par du carbone mort (Bortolami et al., 1973, op. cit.).

Par ailleurs, l'interprétation des teneurs en carbone 14 demande, on l'a vu, la connaissance impérative des teneurs en ${ }^{13} \mathrm{C}$, mais aussi bien souvent les données des mesures de tritium. En effet, dans le rappel des principes, on a évoqué la double origine $\mathrm{du}{ }^{14} \mathrm{C}$. Comme dans le cas du tritium, la balance entre l'apport des essais nucléaires et surtout ther- 


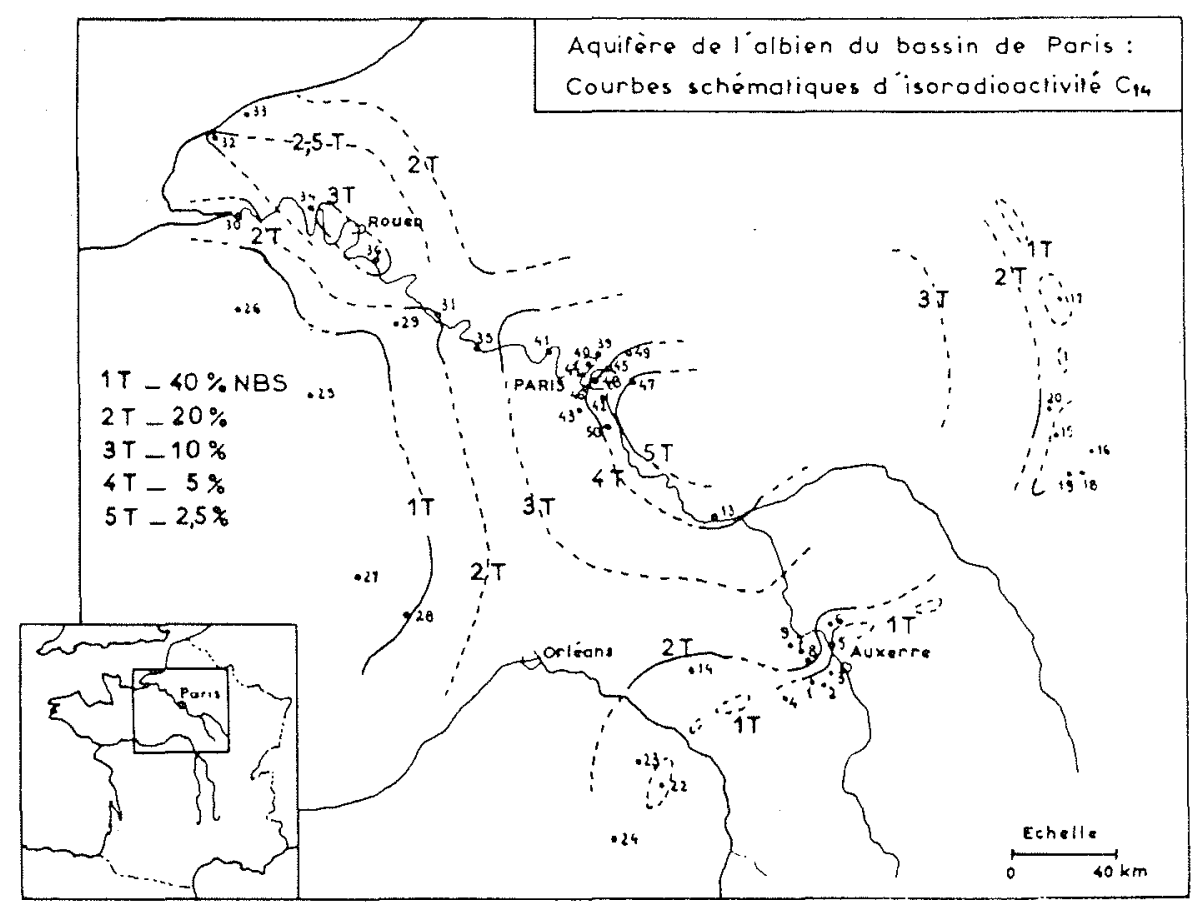

16/Exemple d'évaluation de l'"âge" des eaux souterraines : la nappe de I'Albien du Bassin de Paris.

Les activités du carbone dissous ont été transcrites en termes de temps, exprimés ici en unités de période radio-active du ${ }^{14} \mathrm{C}$ soit 5730 ans, en considérant que l'activité initiale est restée constante et égale à $80 \%$ pour tous les échantillons analysés. Forts de cette simple hypothèse, les auteurs proposent les "ages" compris entre

I'Actuel et environ 5 périodes de ${ }^{4} \mathrm{C}$ pour les eaux captives de la nappe de l'Albien. D'après Evin et Vuillaume (1970).

monucléaires et l'absorption du $\mathrm{CO}_{2}$ dans le cycle photosynthétique, a conduit à un excès variable de ${ }^{14} \mathrm{C}$ dans l'atmosphère. L'évolution des teneurs atmosphériques en ${ }^{14} \mathrm{C}$ est assez comparable à celle du tritium dans les précipitations (fig. 17). Si l'on considère que le passage du ${ }^{14} \mathrm{C}$ par la biosphère avant son entrée dans les eaux souterraines entraine un déphasage d'environ 2 ans (Thilo et Münnich, 1970, op. cit) les teneurs en ${ }^{11} \mathrm{C}$ artificiel seront, en principe, liees aux teneurs en tritium. Ainsi, la présence de tritium permettra

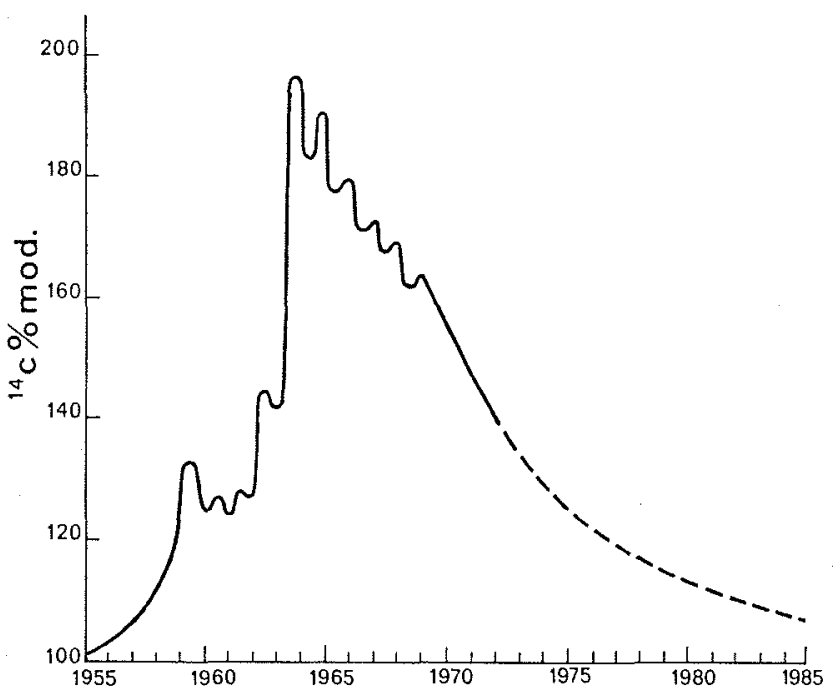

17/ L'excès de radiožarbone apporté par les essais nucléaires.

A l'égal du tritium, le ${ }^{14} \mathrm{C}$ injecté dans la stratosphère est délesté dans les basses couches sous forme de $\mathrm{CO}_{2}$. L'allure générale des variations de teneur représente un traçage du milieu supplémentaire qui peut par exemple étre confronté aux données du tritium dans l'étude des nappes libres. D'après Nydal et al. (1971). de déceler les contaminations par les eaux récentes qui rendent évidemment encore plus délicate l'interprétation des teneurs en ${ }^{14} \mathrm{C}$. D’un autre côté cependant, la présence de carbone 14 artificiel doté d'une fonction d'entrée comparable à celle du tritium présente au contraire un intérêt supplémentaire pour l'étude des nappes libres. En ce cas la décroissance radioactive est bien entendu parfaitement négligeable devant les temps de séjour moyens et le radio-carbone propose alors une équation supplémentaire pour les modèles de mélanges et de circulation.

\subsubsection{Isotopes du soufre}

\subsubsection{Variations naturelles et principes d'utilisation.}

Aux températures biologiques, l'activité bactérienne est la cause première des ségrégations isotopiques entre les espèces stables les plus fréquentes du soufre ("lourd": ${ }^{34} \mathrm{~S}$; "léger" : ${ }^{32} \mathrm{~S}$ ). Comme de règle pour tous les isotopes des éléments métabolisables, cf. (A.3.3.1.3), la biosynthèse fait préférentiellement appel aux isotopes légers. La fraction non métabolisée de la réserve initiale s'en trouve enrichie en isotopes lourds. Dans le cas du soufre, on peut considérer que la composition isotopique $\delta^{34} \mathrm{~S}\left({ }^{1}\right)$ du sulfate du réservoir marin correspond à la sommation de différents effets qui se compensent, c'est-à-dire à un état stationnaire.

On aura donc:

$$
\delta^{34} \mathrm{~S}\left(\mathrm{SO}_{4}^{=} \text {marin }\right)=\text { constant }=+20,3 \% \text { vs } \mathrm{CD}
$$

(1) Pour le soufre, le zéro de l'échelle relative $\delta$ correspond à la teneur en ${ }^{34} \mathrm{~S}$ d'un sulfure (troïlite) provenant d'une météorite précipitée à Canon Diablo (New-Mexico, U.S.A.). L'étalon est ainsi dénommée "Canon Diablo : CD". 
Dans la littérature, les autres domaines de variation se définissent comme suit (voir Rightmire et al. 1974):

$$
\begin{aligned}
& \delta^{34} \mathrm{~S} \text { (évaporites anciennes) }=+8 \grave{a}+32 \\
& \delta^{34} \mathrm{~S}\left(\mathrm{SO}_{4}^{=}, \text {précipitations) }=-1,5 \grave{\mathrm{a}}+19,4\right. \\
& \delta^{34} \mathrm{~S}\left(\mathrm{H}_{2} \mathrm{~S} \text { biogenique }=-35 \grave{\mathrm{a}}+4,0\right. \\
& \delta^{34} \mathrm{~S} \text { (sulfures sédimentaires) }=-35 \grave{\mathrm{a}}+42
\end{aligned}
$$

Compte tenu de la précision des mesures $(\simeq 0,2 \%$ ) les variations naturelles sont confortables et la méthode est fort sensible. L'analyse isotopique du soufre dissous permet dans une large mesure d'en préciser l'origine aux prix de certaines précautions dans l'usage de la technique. Les conditions de collection doivent éliminer toute possibilité de réduction ou d'oxydation postérieure au prélévement par blocage des réactions. Les variations de teneur en ${ }^{34} \mathrm{~S}$ doivent être confrontées à des paramètres chimiques : teneurs totales en $\mathrm{SO}_{4}^{2-}$, rapport $\mathrm{Cl}^{-} / \mathrm{SO}_{4}^{2-}, \mathrm{S}^{2-} / \mathrm{SO}_{4}^{2-}$.

\subsubsection{Acquis et perspectives.}

Les études, encore rares, sont cependant prometteuses. Dans laquifère captif de Floride, Rightmire et al (1974, op. cit.) distinguent entre différentes sources de sulfate dissous : précipitations maritimes chargées en sulfate, mise en solution de gypse de l'aquifère, et mélange avec des eaux marines. Dans l'aquifère Edwards au Texas, ces mêmes auteurs mettent en évidence l'apport éolien de gypse permien à la surface de la zone de recharge et la remontée de sulfures profonds liés à des saumures.

Dans le Latium, Zuppi et al., 1974, op. cit. interprétent les variations de teneurs en ${ }^{34} \mathrm{~S}$ (et oxygène 18 ) des sulfates comme le produit de deux phénomènes : répartition d'une réserve initiale de soufre entre $\mathrm{S}^{=}$et $\mathrm{SO}_{4}^{2-}$ en fonction des potentiels redox du milieu et mélange entre un soufre de type sédimentaire et un soufre profond périvolcanique.

Les possibilités d'exploitation à des fins hydrologiques des variations de teneur en ${ }^{34} \mathrm{~S}$ sont également intéressantes pour les enquêtes sur les circuits chauds. Les formes réduites et oxydées du soufre entrent en réaction chimique et isotopique et l'écart entre les teneurs respectives en ${ }^{34} \mathrm{~S}$ est alors une fonction de la température de réaction. C'est le principe d'un géothermomètre.

\subsubsection{Isotopes de l'azote}

\subsubsection{Variations naturelles du rapport ${ }^{15} \mathrm{~N} /{ }^{14} \mathrm{~N}$.}

Les nombreux degrès d'oxydation possibles de l'azote $-3,0,+3,+5$, les conditions souvent métaboliques de son transfert d'un point à l'autre de son cycle laissent pronostiquer un déterminisme complexe des variations de teneur en isotopes lourds.

Les recherches exploratoires (voir Mariotti et Letolle 1976; Mariotti et al. 1976) indiqueraient que les variations de teneur en ${ }^{15} \mathrm{~N}$ des nitrates des eaux peuvent être schématisées comme la sommation de deux effets :

- mélanges en provenance de deux réservoirs en état stationnaire et donc à composition isotopique constante :

$$
\text { -atmosphère } \delta^{15} \mathrm{~N}=0 \% \text { (1) }
$$$$
- \text { complexe organique des sols } \delta^{15} \mathrm{~N} \simeq+4 \grave{a}+10 \%
$$

- biodégradation partielle des composés ammoniaqués comparable à une distillation partielle et se traduisant par le

(1) L'étalon de l'échelle relative $\delta$ est précisément la composition isotopique moyenne réputée constante de l'azote atmosphérique en tous points de la troposphère. départ d'une "tête" pauvre en ${ }^{15} \mathrm{~N}$ (effet enzymatique) sous forme $\mathrm{NH}_{3}$, et la constitution d'un résidu non métabolisé riche en ${ }^{15} \mathrm{~N}$.

\subsubsection{Interêt hydrologique.}

De nos jours et dans la plupart des régions de culture, la voie d'entrée principale de l'azote atmosphèrique dans les cycles hydrologiques passe par la fertilisation nitrique (les engrais nitriques provenant d'une réaction initiale d'oxydation de l'azote de l'air, au cours de laquelle la composition isotopique $\delta^{15} \mathrm{~N}$ demeure préservée : $\delta^{15} \mathrm{~N}$ fertilisants $\simeq 0$ ).

Le mélange des nitrates du complexe organique des sols $\left(\delta^{15} \mathrm{~N}=+4 \grave{a}+10\right.$ avec $+8 \grave{a}+10$ pour les valeurs les plus fréquentes pour les sols de grande culture) avec les fertilisants nitriques non métabolisés sera donc mis en évidence par la composition isotopique de l'azote nitrique total qui s'inscrira à une valeur intermédiaire.

Les apports ammoniacaux soumis à fermentation avant oxydation en nitrates sont essentiellement imputables aux déchets animaux et humains. Leur contribution à l'azote nitrique dissous sera détectée par l'élévation, parfois considérable $(+20$ à $+30 \%$ ) des teneurs en ${ }^{15} \mathrm{~N}$.

Il est clair que cette nouvelle technique est appelée à connaitre de larges développement pour les investigations sur l'origine des teneurs en azote des eaux souterraines et de surface.

\section{Conclusion}

Le traçage par les isotopes du milieu constitutifs de la molécule d'eau : oxygène 18 , deuterium, tritium, offre à l'hydrologue un outil supplémentaire doté de caractéristiques originales:

-.. Fonction entrée généralement en régime permanent

- Marquage intime des molécules

- Indépendance du traçage initial vis-à-vis des réactions chimiques dans le cas des circuits froids

Possibilité d'utilisation autonome.

Ces propriétés font des isotopes constitutifs un ensemble bien adapté à la reconstitution de l'origine et de l'histoire des eaux au cours de leurs cycles.

Le traçage par les isotopes du milieu dissous est fort différent et s'apparente à un traçage chimique. L'interprétation qui doit faire appel à la connaissance des conditions physicochimiques du milieu, autorise la reconstitution de "l'âge" du carbone dissous $\left({ }^{14} \mathrm{C},{ }^{13} \mathrm{C}\right)$, de l'origine du soufre $\left({ }^{34} \mathrm{~S}\right)$ et de l'azote $\left({ }^{15} \mathrm{~N}\right)$

Les perspectives d'utilisation du traçage par les isotopes du milieu seront peut-être étendues à d'autres éléments comme le silicium, l'argon ou le krypton qui comportent des isotopes marqueurs de temps $\left({ }^{32} \mathrm{Si}, \tau \cong 500\right.$ ans $;{ }^{39} \mathrm{~A}, \tau=270$ ans ${ }^{85} \mathrm{Kr}, \tau=10,4$ ans)

Enfin on doit tenir compte du fait que la concentration croissante dans toute l'hydrosphère de certains composés industriels inertes comme le fréon que l'on dose facilement par une méthode nucléaire, peut à l'avenir constituer un autre traceur du milieu. 


\section{Bibliographie}

ALLISON (G.B.), HUGUES (M.W.) - Environmental tritium in the unsaturated zone : Estimation of recharge to an unconfined aquifer. In "Isotope Techniques in ground wayer Hydrology 1974". Proceed. Symp., I.A.E.A., Vienna, vol. I, 169-189. 1974

BLAVOUX (B.) -- Le problème de la localisation des aires d'alimentation sur la base des gradients isotopiques en altitude. Résumé C.R.Réun. Ann. Sc. Ter., Paris, Soc. Geol. Fr., p. 57. 1976.

BRADLEY (E), FONTES (J.CH.) et ROCHE (M.A.) (en préparation) - Isotope hycirology of the phreatic aquifer of lake rchad.

BIGELEISEN (J.) - Chemistry of isotopes. Science, 147, pp. 463-471. 1965.

Bortolami (G.), FONTES(J.CH.), PANICHI (C.) - Isotopes du milieu et circulations dans les aquifères du sous-sol vénitien. Earth Planet. Sc. Lett., 19, $\mathrm{n}^{\circ}$ 2, 154-167. 1973.

BOTTINGA (Y.), CRAIG (H.) - Oxygen isotope fractionation between $\mathrm{CO}_{2}$ and water and the isotopic composition of marine atmosphere. Earth Planet. Sc. Lett., 5, 285-295. 1969

B Rown (R.M.) -- Hydrology of tritium in the Ottawa valley. Geochim. Cosmochim. Acta, 21, 199-216, 1961.

BROWN (R.H.) - Distribution of hydrogen isotopes in Canadian waters. In "Isotope Hydrology 1970" I.A.E.A., Vienna, 3-21. 1970.

BROWN (L.J.), TAYLOR (C.B.) - Geohydrology of the Kaikoura plain, Marlborough, New-Zealand. In "Isotope techniques in groundwater Hydrology 1974". Proceed. Symp., I.A.E.A., Vienna, vol. 1, 169$189,1974$.

CRAIG (H.) -- Standards for reporting concentrations of deuterium and oxygen-18 in natural waters. Science, 133, 1833-1834, 1961 a.

CRAIG (H.) - Isotopic variations in meteoric waters. Science, 133, 1833-1834, $1961 \mathrm{~b}$.

CRAIG (H.), GORDON (L.I.), HORIBE (Y.) - Isotopic exchange effects in the evaporation of water:I low temperature experimental results. J. Geophys. Res., 68, 5079-5087, 1963.

CRAIG (H.) - The isotopic geochemistry of water and carbon in geothermal areas. In "Nuclear Geology in Geothermal Areas", CNR Laboratorio di Geologia Nucleare, Pisa, 17-53, 1963.

CRAIG (H.), GORDON (L.I.) - Deuterium and oxygen-1 8 variations in the ocean and marine atmosphere. In "Stable Isotopes in Oceanographic Studies and Paleotemperatures". CNR - Laboratorio di Geologia Nucleare, Pisa, 9-130, 1965

CRAIG (H.) - Isotopic composition and origin of the Red Sea and Salton Sea Geothermal brines. Science, 154, 1544-1548, 1966.

DAMON (P.E.), LONG (A.), GREY (D.) - Fluctuations of atmospheric during the last six millennia. J. Geophys. Res., 71, 1055-1063, 1966.

DANSGAARD (W.) - Stable isotopes in precipitation. Tellus, 19, 435$468,1964$.

DAvis (G.H.), PAYNe (B.R.), DinCER (T.), FloRKoWSKI (T.), GATTINGER (T.) - Seasonal variations in the tritium content of ground-waters of the Vienna Basin, Austria, In : "Isotopes in $\mathrm{Hy}$. drology", I.A.E.A. Vienna 1967, 451-473, 1967.

EHALT (D.) KNOTT (K.) -- Kinetische Isotopentrennung bei der Verdampfung von Wasser. Tellus, 17, 389-397.1965.

EVIN (J.) VUillaUmE (Y.) -- Etude par le radiocarbone de la nappe captive de l'Albien du Bassin de Paris. In : "Isotope Hydrology 1970", Proceed. Symp. I.A.E.A., Vienna, 315-332, 1970.

FErRara (G.C.), Gonmintini (R.), Panichi (C.) - La composizione isotopica del vapore di alcuni soffioni di Larderello e dell' aqua di alcuni sorgenti e mofete della Toscana. Atti Soc. Tosc. Sci. Nat., 72, 570-588, 1965.

FONTES (J.C.), GONFIANTINI (R.) -- Comportement isotopique au cours de l'évaporation de deux bassins Sahariens. Earth Plan. Sci. Lett., 3, 258-266, 1967.

FONTES (J.C.) - Quelques aspects isotopiques du cycle des eaux naturelles. C.R. Coll. Int. Isot. Oxygène, European Molecular Biology Organization, CEA, Cadarache 1972, 197-225, 1974.

FONTES (J.C.), OLIVRY (J.C.) - Gradient isotopique entre 0 et $4000 \mathrm{~m}$ dans les précipitations du Mont Camesuun. Résumé C.R. Réun. Ann. Sc. Ter., Soc. Géol. Fr., Paris, p. 171, 1976.
FONTES (J.CH), GARNIER (J.M.) -.. COrrection des activités apparentes en ${ }^{14} \mathrm{C}$ du carbone dissous : estimation de la vitesse des eaux en nappes captives. Résumé. C.R. Réun. Ann. Sc. Ter., Paris, Soc., Géol. Fr, p. 170, 1976.

GAI (J.R.) - Environmental isotope balance of Lake Tiberias. In : "Isotope Hydrology 1970", I.A.E.A., Vienna, 109-127. 1970

GONFIANTINI (R.), GRATZIU (S.), TONGIORGI (E.) - Oxygen isotopic composition of water in leaves. In : "Isotopes and radiation in soil plant nutrition studies". Proceed. Symp., I.A.E.A., Vienna, $405-410,1965$.

GONFIANTINI (R.) - Effetti isotopici nell'evaporazione di acque salate. Atti Soc. Tosc. Sci. Nat., $72 ; 550-569.1965$.

GONEIANTINI (R.) - Notes on isotopes Hydrology. I.A.E.A. Vienna. 45 pp. Multigraph, 1971.

GONFIANTI (R.), BORSI (S.), FERRARA (G.), PANICHI (C.) - Isotopic composition of waters from the Danakil depression (Ethiopia). Earth Planet. Sci. Lett., 18, n 1, 13-21, 1973.

INTERNATIONAL ATOMIC ENERGY AGENCY - Environmental Isotope Data $\mathrm{n}^{\circ} 1$ : World Survey of Isotope Concentration in Precipitation (1953-1963), Tech. Rep. Ser, n 96, I.A.E.A., Vienna, 1969.

INTERNATIONAL ATOMIC ENERGY AGENCY - Envíronmental isotope data $n^{\circ} 2$ : World Survey of Isotope Concentration in Precipitation (1962-1964), Tech. Rep. Ser. n 117, 402 pp., I.A.E.A., Vienna., 1970;

INTERNATIONAL ATOMIC ENERGY AGENCY - Environmental isotope data $\mathrm{n}^{\circ} 3$ : World Survey of Isotope Concentration in Precipitation (1960-1967), Tech. Rep. Ser. n 129,402 pp., I.A.E.A., Vienna. 1971;

INTERNATIONAL ATOMIC ENERGY AGENCY - Environmental isotope data $n^{\circ} 4$ : World Survey of Isotope Concentration in Precipitation (1968-1969), Tech. Rep. Ser., n 147, 334 pp., I.A.E.A., Vienna. 1973.

LAL (D.), SUESS (H.E.) - The radioactivity of the atmosphere and hydrosphere. An. Rev. of Nucl. Sci, 18, 407-437, 1968.

LESAint (C.), MERLIVAT (L.), BRICOUT (J.), FONTES (J.CH.), GAUTHERET (R.) - Sur la composition en isotopes stables de l'eau de la Tomate et du Mais. C.R.Acad. Sc. Paris, 278, 2925$2930,1974$.

MARgRita (R.), EVIN (J.), FlandRIN (J.), PAloc (H.) - Contribution des mesures isotopiques à l'étude de la Fontaine de Vaucluse. In : "Isotope Hydrology 1970" Proceed. Symp., I.A.E.A., Vienna, 333-348, 1970.

Mariotti (A.), Ben Halima (A.), BERGER (G.) - Apport de l'étude isotopique de l'azote à la connaissance de la pollution des aquifères souterrains par les nitrates, en milieu agricole (Brie, Beauce France). Revue de Géographie Physique et Géologie Dynamique (sous-presse), 1976.

MARIOTti (A.), LEtolle (R.) -- Application de l'étude isotopique de l'azote en hydrologie et hydrogéologie. Analyse des résultats ob tenus sur un exemple précis : le bassin de Mélarchez (Seine et Marne, France). Journal of Hydrology (sous-presse). 1976.

MERLIVAT (L.), NIEF (G.) - Fractionnement isotopique lors des changement d'état solide-vapeur et liquide-vapeur de l'eau à des températures inférieures à $0^{\circ} \mathrm{C}$. Tellus, 19, 122-127. 1967.

MERLIVAT (L.) -- L'étude quantitative de bilans de lacs à l'aide des centrations en deuterium et oxygène-18 dans l'eau. In: "Isotope Hydrology 1970", , I.A.E.A., Vienna, 89-107, 1970.

MOOK (W.G.) - On the reconstruction of the initial ${ }^{14} \mathrm{C}$ content of groundwater from the chemical and isotopic composition. Proceed. 8 th Int. Conf. on radiocarbon dating. Wellington, N.Z., D. 31-41. 1972.

NIEF (C.), BOTTER (R.) - Analyse de composés hydrogénés simples au spectrométre de masse. In "Advances in Mass Spectrometry. Proceed. Symp. J.D. Waldron ed. Pergamon Press, 515-525, 1960.

NIR (A.) - On the interpretation of tritium "age" measurements of ground-water. J. Geophys. Res., 69, 2589-2595, 1964.

NIR (A.) - Development of Isotope Methods Applied to Ground-water Hydrology. In : "Isotope Techniques in the Hydrologic Cycle". Monograph. $\mathrm{n}^{\circ} 11$, Am. Geophys. Union, Washington D.C., 109$116,1967$.

OLIVE (PH.) - Contribution à l'étude géodynamique du cycle de l'eau dans l'hémisphère nord par la méthode du tritium. Thèse, Paris, $138 \mathrm{p}, 1970$. 
O'NEIL (J.R.) - Hydrogen and oxygen isotope fractionation between ice and water. J. Phys. Chem., 72, 3683-3864. 1968.

PAYNE (B.R.), YURTSEVER (Y.) - Environmental isotopes as a hydrogeological tool in Nicaragua. In : "Isotope Technique in groundwater Hydrology, 1974". Proceed. Symp., I.A.E.A., Vienna, vol. I, 193-202, 1974

PEARSON (F.J.) Jr, HANSHAW (B.B.) - Sources of dissolved carbonate species in ground-water and their effects on carbon-14 dating. In "Isotope Hydrology 1970", I.A.E.A., Vienna, 271-286, 1970.

PEARSON (F.J.) JT, BEDINGER (M.S.), JONES (B.F.) - Carbon-14 ages of water from the Arkansas springs. Proceed. 8th Int. Conf. on radiocarbon dating, Wellington, N.Z., D, 19-30, 1972.

PEARSON (F.J.) Jr, SWARZENKI $-{ }^{14} \mathrm{C}$ evidence for the origin of arid region groundwater, Northeastern province Kenya, in : "Isotope techniques in ground-water Hydrology 1974" Proceed. Symp. I.A.E.A., Vienna, Vol. II, 95-109, 1974.

RIGHTMIRE (C.T.), PEARSON (F.J.), Jr, BACK (W.), RYE (R.O.) and HANSHAW (B.B.) - Distribution of sulfur isotopes of sulfates in groudwaters from the principal artesian aquifer of Florida and the Edwards aquifer of Texas, United States of America : in Isotope Techniques in Groundwater Hydrology, v.II, IAEA, Vienna, p. 191-207, 1974.

SMith (D.B.), WEARN (P.L.), Richards (H.J.), Rowe (P.C.) -Water movement in the unsaturated zone of high and low permeability strata by measuring natural tritium. In : "Isotope Hydro logy 1970". I.A.E.A., Vienna, 73-87. 1970.

SUESS H.E. - Tritium geophysics as an international research project. Science, 163, 1405-1410, 1969.

TAMERS (M.A.) - Surface water infiltration and groundwater movement in arid zones of Venezuela. In : "Isotopes in Hydrology". Proceed. Symp., I.A.E.A., Vienna, 339-353.1967.
TAMERS (M.A.) SCHARPENSEEL (H.W.) - Sequential sampling of radiocarbon in groundwater. In : "Isotope hydrology 1970". Proceed. Symp., I.A.E.A., Vienna, 241-257, 1970.

TAMERS (M.A.), STIPP (J.J.), WEINER (R.) - Radiocarbon ages of groundwater as a basis for the determination of safe limits of aquifer exploitation. Environmental Research, 9, 250-264, 1975.

THATCHER (L.L.) PAYNE (B.R.) - The distribution of tritium in precipitation over continents and its significance to groundwater dating. Proceed. 6th Int. Conf. Radiocarbon and Tritium Dating, Pullman, Washington, D.C., 604, 1965

THILO (L.), MUNNICH (K.O.) - Reliability of carbon-14 dating of groundwater : effect of carbonate exchange. In : "Isotope Hydrology 1970", I.A.E.A., Vienna, 259-270, 1970.

UREY (H.C. - The thermodynamic properties of isotopic substances. $J$. Chem. Soc. (London), 562-581. 1947

VOGEL (J.C.) - Investigation of groundwater flow with radiocarbon. In : "Isotopes in Hydrology", Proceed. Symp., I.A.E.A., Vienna, $355-369,1967$

VOGEL (J.C.) - Carbon-14 dating of groundwater. In : "Isotope Hydrology 1970". Proceed. Symp., I.A.E.A., Vienna, 225-239, 1970.

VOGEL (J.C.), VAN DIJKEN (M.) - Determination of groundwater recharge with tritium. Proceed. Symp. Atomic Energy Board. Pelindaba, Pretoria, 1972.

ZIMMERMAN (U.), EHHALT (D.H.) - Stable isotopes in study of water balance of Lake Neusiedl, Austria : investigation of the reliability of the stable isotope method. In : "Isotope Hydrology 1970", I.A.E.A., Vienna, 129-138, 1970

Zuppi (G.M.), Fontes (J.CH.), LETOLLE (R.) - Isotopes du milieu et circulations d'eaux sulfurées dans le Latium. In : "Isotope Technique in groundwater Hydrology 1974". Proceed. Symp. I.A.E.A., Vienna, vol. 1. 341-361, 1974

\section{Discussion}

Président : M. Pierre LEVEQUE:

M. Le Président remercie M. Fon tes et M. le Professeur Le rolle de leurs intéressants exposés, vivant reflet des plus récentes recherches en la matière.

Il ouvre ensuite la discussion en posant la question suivante :

"N'êtes-vous jamais gêné au cours de vos expériences par les retombées des explosions thermonucléaires?"

Non au contraire ! répond M. FONTES ; malheureusement pour les géologues, du fait de la cessation des explosions nucléaires dans l'atmosphère, les teneurs en tritium dues à ces dernières diminuent de plus en plus ; on tend à remplacer cet élément par le krypton 85 qui a une période voisine de celle du tritium ( 8 à 10 ans) et dont la concentration va croissant car c'est l'un des effluents rejetés par les réacteurs nucléaires.

M. Paul Ch. LEV EQUE intervient ensuite en ces termes:
"M. FONTES a parlé des traceurs naturellement mélangés aux eaux de pluie et à l'atmosphère, mais, dans le domaine des nappes souterraines, les réacteurs nucléaires vont aussi, semble-t-il, nous rendre service ; en effet, il y a presque toujours d'assez grandes quantités de tritium évacuées par les systèmes de refroidissement des réacteurs nucléaires, et nous comptons beaucoup sur cet apport (qui est largement au-dessous des normes de sécurité, bien entendu) pour nous apporter des renseignements qui commençaient à nous faire défaut en raison de l'abaissement de la teneur en tritium des précipitations".

Merci de votre intervention, dit M. Le Président. Il me reste à remercier tous ceux qui ont participé, ce matin, soit aux exposés, soit aux discussions, et qui ont rendu cette réunion particulièrement intéressante.

La séance est levée à $12 \mathrm{~h} 30$. 